\title{
Plant NLR targets P-type ATPase for executing plasma membrane depolarization leading to calcium influx and cell death
}

\author{
Hye-Young Lee ${ }^{1} \dagger$, Ye-Eun Seo ${ }^{1,2} \dagger$, Joo Hyun Lee ${ }^{1}$, So Eui Lee ${ }^{1,2}$, Soohyun $\mathrm{Oh}^{1,2}$, Jihyun \\ $\mathrm{Kim}^{1,2}$, Seungmee Jung ${ }^{1}$, Haeun Kim ${ }^{1,2}$, Hyojeong Park ${ }^{1,2}$, Sejun Kim ${ }^{1,2}$, Hyunggon Mang ${ }^{1 *}$, \\ and Doil Choi ${ }^{1,2 *}$
}

\author{
Affiliations: \\ ${ }^{1}$ Plant Immunity Research Center, Seoul National University, Seoul, 08826, Republic of Korea \\ ${ }^{2}$ Department of Plant Science, Plant Genomics and Breeding Institute, Seoul National University, Seoul, 08826, \\ Republic of Korea \\ *Correspondence to: doil@snu.ac.kr (D.C.); skymang6@snu.ac.kr (H.M.) \\ †These authors contributed equally to this work.
}

\begin{abstract}
Hypersensitive response (HR) is a robust immune response mediated by plant nucleotide-binding and leucine-rich repeat receptor (NLR). However, the early molecular event linking NLR to cell death is obscure. Here we demonstrate that NLR targets plasma membrane $\mathrm{H}^{+}$-ATPases (PMA) generating electrochemical potential across the membrane. CC $^{\mathrm{A}} 309$, an autoactive N-terminal domain of pepper coiled-coil NLR (CNL), associates with PMAs and its autoactivity is affected by silencing or overexpression of PMA. CC ${ }^{A} 309$-induced extracellular alkalization accompanied with membrane depolarization is followed by calcium influx and cell death. CC ${ }^{\text {A } 309}$ interacts with $\mathrm{C}$-terminal regulatory domain of PMA and 14-3-3 negatively affects $\mathrm{CC}^{\mathrm{A}} 309$-induced cell death. Moreover, pharmacological experiments with fusicoccin, an irreversible PMA activator, confirmed that CC- and CNL-mediated cell death occurred through inhibiting PMA. We propose PMAs as the primary target of plasma membrane-associated CNL to disrupt electrochemical homeostasis leading to $\mathrm{HR}$ cell death.
\end{abstract}

\section{Main text}

Plants rely on the innate immune system against pathogen challenges. The immune responses are provoked by the perception of pathogen infection via the cytosolic immune receptors, designated nucleotide-binding and leucine-rich repeat receptor $(\mathrm{NLR})^{1}$. Plant NLRs are subdivided into two major classes based on their N-terminal domain; coiled-coil NLR (CNL) and Toll interleukin-1 receptor NLR $(\mathrm{TNL})^{2}$. The NLRs are activated upon the recognition of pathogenic effectors, resulting in defense responses including hypersensitive response (HR), a form of programmed cell death ${ }^{3}$. Although HR is considered as one of the most robust defense responses and facilitates restriction of pathogen growth in the infected cells, the underlying mechanisms are elusive.

Recent remarkable discoveries shed light on the molecular events of NLR-mediated cell death. Like bacterial and mammalian TIR domains, plant TIR domain in TNL cleaves $\mathrm{NAD}^{+}$, leading to cell death signal transduction ${ }^{4,5}$. Moreover, structural and biochemical characterization of Arabidopsis ZAR1 revealed that the $\mathrm{N}$-terminal $\alpha$-helix of CC domain in the activated resistosome forms a funnel-shaped structure, which is essential for ZAR1-mediated cell death $^{6}$. However, the early molecular events in the onset of cell death by activated NLR remain to be elucidated.

Previously, we reported an Autonomous and Ancient NLR group (ANL) comprising CC domains conferring HR-mimic cell death in seed plants ${ }^{7}$. To take experimental advantages of small size and simple structure, CC ${ }^{\mathrm{A}} 309$, an autoactive CC domain in pepper ANL, was adopted to identify novel immune components underlying NLR-mediated cell death.

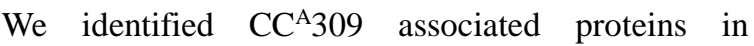
Nicotiana benthamiana by in planta coimmunoprecipitation (co-IP) followed by liquid chromatography-tandem mass spectrometry (Extended Data Table 1). Since CC $\mathrm{CC}^{\mathrm{A}} 309$ is localized on plasma membrane (PM), plasma membrane $\mathrm{H}^{+}$ATPase3 (PMA3) among the candidates was selected for further experiments. PMAs are active proton pumps to establish the electrochemical gradient across the PM which is essential not only for the exchange of ions and solutes across the PM but also for cell viability as shown by genetic analysis in Arabidopsis and yeast ${ }^{8,9}$. Co-IP and bimolecular fluorescence complementation (BiFC) assays validated that PMA3 
associates with CC ${ }^{\mathrm{A}} 309$ but not NbRIN4, a homolog of PM-localized Arabidopsis RPM1-interacting protein 4, indicating that PMA3 may specifically interact with CC ${ }^{\mathrm{A}} 309$ (Fig. 1a, b, Extended Data Fig. 1). The $C^{A} 309$-induced cell death but not Bax encoding human BCL2-associated X was significantly enhanced in PMA3-silenced plants (Fig. 1c, Extended Data Fig. 2) and compromised by transient expression PMA3 in $N$. benthamiana (Fig. 1d). These results suggest that PMA3 plays an important role in

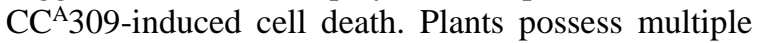
isoforms of differentially expressed PMAs with functional redundancy ${ }^{8}$. We identified additional 12 putative PMAs from $N$. benthamiana genome ${ }^{10}$ and further revealed that PMA1 in the same phylogenetic clade of PMA3 and PMA4 in another clade also interacted with $\mathrm{CC}^{\mathrm{A}} 309$, suggesting that $\mathrm{CC}^{\mathrm{A}} 309$ associates with multiple PMAs in planta (Extended Data Fig. 3, 4). Consistent with these data, multiple PMA-silenced plants (PMA1/2/3) showed significantly enhanced cell death caused by $\mathrm{CC}^{\mathrm{A}} 309$, note that $P M A 1 / 2 / 3 / 4$-silenced plants were not subjected to the test because of extremely abnormal growth (Extended Data Fig. 5).

As PMA is a major $\mathrm{H}^{+}$pump in the plasma membrane, we monitored the $\mathrm{pH}$ change in the apoplast and cytoplasm during $\mathrm{CC}^{\mathrm{A}} 309$-induced cell death. To measure $\mathrm{pH}$ values inside and outside of the $\mathrm{PM}$, we utilized the ratiometric pHluorin sensors, PM-CYTO

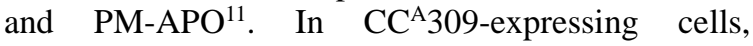
apoplastic $\mathrm{pH}$ was increased $(\approx \mathrm{pH} 0.5)$, while cytoplasmic $\mathrm{pH}$ was reduced $(\approx \mathrm{pH} 0.54)$, suggesting that the electrochemical potential across the PM is disturbed in the progress of $\mathrm{CC}^{\mathrm{A}} 309$-induced cell death (Fig. 2a, Extended Data Fig. 6). However, the $\mathrm{pH}$ change was moderate in PMA3 co-expressing cells (Fig. 2b), implying that $\mathrm{CC}^{\mathrm{A}} 309$ triggers alkalization of extracellular space by inhibition of PMAs. The early responses during HR are known to be followed by the activation of PM-bound ion channels and the assembly of an NADPH oxidase complex for the generation of reactive oxygen species ${ }^{12}$. Moreover, the elevation of cytosolic calcium places upstream of the oxidative burst in NLR-mediated cell death ${ }^{13}$. Thus, we monitored the influx of $\mathrm{Ca}^{2+}$ in cytosol and change of $\mathrm{pH}$ in apoplast during $\mathrm{CC}^{\mathrm{A}} 309$-induced cell death in $N$. benthamiana using cytosolic $\mathrm{Ca}^{2+}$ sensor, YC3.6 and PM-APO, respectively ${ }^{14}$. In the repeated experiments, we observed the elevation of cytosolic calcium ions at 6 8 hrs post-induction of $\mathrm{CC}^{\mathrm{A}} 309$ expression which is significantly later than the elevation of $\mathrm{pH}$ at $2 \sim 4$ hrs in the apoplast (Fig. 2c, Extended Data Fig. 7). As a verification, artificial alkalization of the apoplast also triggered cytosolic $\mathrm{Ca}^{2+}$ accumulation (Fig. 2d). Furthermore, we observed the depolarization of PM by transient expression of CC $\mathrm{C}^{\mathrm{A}} 309$ using DiBAC4 as an indicator ${ }^{15}$ (Fig. 2e). These results indicate that $C^{A} 309$ triggers the alkalization of extracellular space and membrane depolarization via inhibition of PMAs leading to the influx of $\mathrm{Ca}^{2+}$ into the cytosol.

The PMA protein is composed of three major cytosolic domains, including a central catalytic and a C-terminal regulatory domain and is activated by phosphorylation on penultimate threonine allowing for the binding of the activator protein, 14-3-3 resulting in relieve of the autoinhibition state ${ }^{16,17}$. We found that $\mathrm{CC}^{\mathrm{A}} 309$ interacts with both central and C-terminal domains of PMA but a stronger co-IP signal was observed with the C-terminal domain (Fig. 3a).

Because 14-3-3 is associated with both PMA3 and $\mathrm{CC}^{\mathrm{A}} 309$ in our co-IP experiment (Fig. 3b), the roles of 14-3-3 in $\mathrm{CC}^{\mathrm{A}} 309$-induced cell death were further investigated. Interestingly, co-expression of 14-3-3 and $\mathrm{CC}^{\mathrm{A}} 309$ compromised the association between $\mathrm{CC}^{\mathrm{A}} 309$ and PMA3 (Fig. 3c), suggesting that 14-3-3 may have an inhibitory effect on the interaction between PMA3 and $\mathrm{CC}^{\mathrm{A}} 309$. Consistent with co-IP data, significantly enhanced cell death was observed in 14-3-3-silenced plants (Fig. 3d, Extended Data Fig. 8). Next, we determined whether PMA activity indeed affects CC domain-induced cell death. Fusicoccin, an irreversible activator of PMA by inhibiting dissociation of PMA/14-3-3, was used for further experiments ${ }^{18}$. The leaves expressing CC domains were infiltrated with fusicoccin. Surprisingly, only cell death induced by PM-localized and PMA3-associated CC domains (CC ${ }^{\mathrm{A}} 309, \mathrm{CC}^{\mathrm{A}} \mathrm{Pvr} 4$, and $\mathrm{CC}^{\mathrm{NbZAR} 1}$ ) was inhibited by fusicoccin without deteriorative effect on protein expression, but not cytosol-localized $\mathrm{CC}^{\mathrm{R} 3 \mathrm{a}}$ (Fig. 4a,b,c, Extended Data Fig. 9a). To expand our understanding of PMA on resistance protein ( $R$ protein)-mediated cell death, functionally characterized NLRs were subjected to test fusicoccin effect. Full-length NLRs were expressed with or without its cognate effector in $N$. benthamiana and followed by fusicoccin treatment. As results, cell death mediated by PM-localized CNLs (Prf, HRT, Pvr4, RPS2, and NbZAR1 ${ }^{\text {D481V }}$ ) but not non-PMlocalized CNLs (R3a and Rx) and non-PM-localized TNLs (N, SNC1 and RPS4) was compromised by fusicoccin (Fig. 4d, Extended Data Fig. 9b). Moreover, full-length Pvr4 and NbZAR1 interact with PMA3, suggesting that PMA is a pivotal regulator of cell death in PM-associated R proteins (Extended Data Fig. $10)^{19,20,21}$.

PM is the site of recognizing environmental cues and generating the secondary signals by modifications of PM-bounded channels and transporters. Although it is well-studied that the perception of pathogen results in the exchange of ion and solute across the PM as well as generation of reactive oxygen species (ROS), how activated NLR provokes cell death in immune response was obscure ${ }^{22,23}$. Our data clearly indicate that CNLs are associated with and inhibit PMA, 
resulting in alkalization of extracellular space leading to membrane depolarization. We propose PMA as a primary target of membrane-associated CNLs to facilitate both the development of cell death by disturbing homeostasis of membrane potential and the generation of secondary signals such as $\mathrm{Ca}^{2+}$ influx and ROS burst to trigger the defense response and HR cell death in plants (Extended Data Fig. 11).

\section{Methods}

Plant materials and growth conditions

Nicotiana benthamiana were grown in walk-in growth chamber at $25{ }^{\circ} \mathrm{C}$ with a $16 \mathrm{~h} / 8 \mathrm{~h}$ (day/night) cycle. Four-weak-old plants were used in Agrobacteriummediated transient expressions.

\section{Transient gene expression}

For in planta transient overexpression, Agrobacterium tumefaciens strain GV3101 cells carrying the desired binary vector clones were grown overnight at $28^{\circ} \mathrm{C}$ in LB media with appropriate antibiotics. The grown cells were harvested by centrifugation at 3,000 rpm for $10 \mathrm{~min}$ and resuspended into the infiltration buffer (10 mM MES (2-[N-morpholino] ethanesulfonic acid), 10 $\mathrm{mM} \mathrm{MgCl}_{2}$ and $150 \mu \mathrm{M}$ acetosyringone, $\mathrm{pH}$ 5.6). Resuspended cell suspension were incubated for 1 3 $\mathrm{hr}$ at room temperature and pressure-infiltrated with a $1 \mathrm{~mL}$ needless syringe into $N$. benthamiana leaves. To visualize cell death, the leaves were photograph after $3 \sim 5$ days after infiltration using Cy5 and Cy3 channels in Azure 400 (Azure biosystem).

\section{Constructs}

Clones for epitope tagged proteins were generated using ligation-independent cloning method ${ }^{24}$. The inserts for NbRIN4 (Niben101Scf03479g05049.1), Nb14-3-3 (Niben101Scf02367g04001.1), NbPMA1 (Niben101Scf00593g01002.1), N N N N N (Niben101Scf07395g00031.1), N N N N N (Niben101Scf03979g02010.1) were amplified from $N$. benthamiana cDNA and cloned into the pCAMBIA2300-LIC vectors (Cauliflower mosaic virus 35S promoter with $\mathrm{N}$-term 6xHA, C-term eGFP or C-term 3xFLAG tag). For expression of fragments of PMA3, PMA3-N (residue 1-64), PMA3-M (residue 305-650) and PMA3-C (residue 846-956) were cloned into pCAMBIA2300-C-eGFP-LIC vector. For inducible expression, $C^{A} 309$ (nucleotide 1-420) was cloned into XVE-DC-6xMYC vector by gateway cloning (Invitrogen) ${ }^{25}$. To generate single genesilenced plants, the gene-specific fragment of PMA3 (nucleotide 308-474) or 14-3-3 (nucleotide 54-338) was cloned into TRV2-LIC vector. For co-silencing of PMAs (PMA1/2/3/4), the fragments of PMA1 (nucleotide 4-204), PMA2 (nucleotide 202-401), PMA3 (nucleotide 402-601), and PMA4 (nucleotide 599-798) were fused by overlap PCR and cloned into
TRV2-LIC vector. The CC domain of NbZAR1 (nucleotide 1-420), Pvr4 (nucleotide 1-495) or R3a (nucleotide 1-480) was fused to eGFP epitope by cloning to pCAMBIA2300-C-eGFP-LIC vector. The primers used for cloning are listed in Extended Data Table 2.

\section{Virus-induced gene silencing (VIGS)}

VIGS was performed described by Liu et $\mathrm{al}^{26}$. Twoweek-old $N$. benthamiana plants were used in VIGS. A. tumefaciens strain GV3101 harboring TRV1 and TRV2 with corresponding fragments from desired genes were grown overnight at $28{ }^{\circ} \mathrm{C}$ in $\mathrm{LB}$ media. Harvested cells were resuspended into infiltration buffer (10 mM MES, $10 \mathrm{mM} \mathrm{MgCl}_{2}$ and $150 \mu \mathrm{M}$ acetosyringone, pH5.6) to a final O.D $\mathrm{D}_{600}$ of 0.3 . Resuspended cell suspension was incubated for $1 \sim 3 \mathrm{hr}$ at room temperature. Cell suspension of TRV1 and TRV2 were mixed in a 1:1 ratio before infiltration. At two weeks, the upper leaves were used for further experiments. To estimate the efficiency of silencing, transcripts level of genes was validated by quantitative RT-PCR. Total RNA was isolated from leaves of silenced plants using TRI reagent (MRC), and cDNA was synthesized using SuperScript II Reverse Transcriptase (Invitrogen). qRT-PCR was performed using a CFX96 Touch Real-Time PCR Detection Systems (Bio-rad) with SsoAdvanced Universal IT SYBR Green Supermix (Bio-rad). Elongation factor$1 \alpha(E F-1 a)$ was used as an internal standard. Genespecific primers used for expression analysis are listed in Extended Data Table 2.

\section{Co-immunoprecipitation}

Agrobacterium-mediated transient expression in Nicotiana benthamiana was performed as described with some modifications ${ }^{27}$. Briefly, Agrobacterium GV3101 $\left(\mathrm{OD}_{600}=0.5\right)$ carrying different vectors tagged with HA, FLAG, GFP or MYC was syringeinfiltrated into 4-week-old $N$. benthamiana leaves. The infiltrated leaves were collected at 36 hpi for coIP. The GFP tagged proteins were immunoprecipitated with $10 \mu \mathrm{l}$ of $\alpha$-GFP agarose beads (MBL) in $500 \mu \mathrm{l}$ co-IP buffer $(150 \mathrm{mM} \mathrm{NaCl}$, $50 \mathrm{mM}$ Tris-HCl, pH7.5, 2 mM EDTA, 10 mM DTT, $0.2 \%$ Triton X-100, 1:100 cOmplete protease inhibitor cocktail (Roche). A small aliquot of samples in co-IP buffer was used for input control before adding $\alpha$-GFP agarose beads. The co-IP samples were gently rotated for $3 \mathrm{hrs}$ at $4^{\circ} \mathrm{C}$. The beads were collected and washed more than five times with washing buffer (500 mM $\mathrm{NaCl}, 25 \mathrm{mM}$ Tris-HCl, pH7.5, $1 \mathrm{mM}$ EDTA and $0.15 \%$ NP-40. The samples were analyzed by immunoblot with an appropriate antibody.

\section{Bimolecular fluorescence complementation} pCAMBIA2300-LIC vector was modified for cloning BiFC construct to contain YN (residues 1-155 of YFP) or YC (residues 156 to the stop codon of YFP). 
NbRIN4 and CC 309 were fused with $\mathrm{YN}$ at their Cterminal in the pCAMBIA2300-LIC-YN vector. NbPMA3 was fused with YC at its C-terminal in the pCAMBIA2300-LIC-YC vector. NbRIN4-YN or $\mathrm{CC}^{\mathrm{A}} 309-\mathrm{YN}$ was transiently expressed with NbPMA3-YC and p19 silencing suppressor by agroinfiltration at a 1:1:1 ratio in $N$. benthamiana leaves. Then, treated with $2 \mathrm{mM} \mathrm{LaCl}$ at 16 hpi to inhibit CC $^{\text {A } 309-i n d u c e d ~ c e l l ~ d e a t h . ~ I m a g i n g ~ w a s ~ p e r f o r m e d ~}$ using a confocal microscope (Leica SP8 X) at 2 dpi. To observe the BiFC fluorescence and chlorophyll autofluorescence, 514 and $633 \mathrm{~nm}$ white light laser were excited, respectively, and 525-580 and 650-720 $\mathrm{nm}$ emission wavelengths were used. Images were processed using LAS X software.

\section{Quantification of intensity of cell death}

The intensity of cell death was quantified by measuring chlorophyll fluorescence using a closed FluorCam (Photon Systems Instruments) and Fluorcam 7.0 software. The detached leaves were exposed to the super pulse in the closed chamber and minimum fluorescence (F0), maximum fluorescence (Fm) and maximum quantum yield of photosystem II (Fv/Fm) parameters were determined by default $\mathrm{F}$. $\mathrm{v} / \mathrm{Fm}$ protocol of system. The Fv/Fm value of empty vector-infiltrated leaves was around 0.77 .

\section{Phylogenetic tree analysis}

Amino acid sequences of PMAs in Arabidopsis thaliana, $N$. benthamiana and $N$. plumbaginifolia were aligned using Muscle ${ }^{28}$, and the alignment was used to construct a phylogenetic tree with the Maximum Likelihood method using MEGA ${ }^{29}$. Evolutionary distances were computed using the JTT matrix- based method with bootstrap test (500 replicates).

\section{Fusicoccin treatment}

Fusicoccin (1 mM, Sigma-Aldrich F0537) stock solution was prepared in ethanol and diluted in distilled water to $1 \mu \mathrm{M}$. After $12-16 \mathrm{hpi}, 1 \mu \mathrm{M}$ fusicoccin or mock ( $0.1 \%$ ethanol) were infiltrated into the leaf region that has been infiltrated with Agrobacteria for transient expression of indicated genes. To determine if fusicoccin inhibits $\mathrm{R}$ proteinmediated cell death, Pto/AvrPto (for activation of Prf), HRT/TCV-CP, Pvr4/PepMoV-NIb, full-length of $R P S 2, N b Z A R 1^{D 481 V}$ (autoactive form of NbZAR1), $N / p 50$, full-length of $S N C 1$, full-length of RPS4, $R 3 a / A v r 3 a$ and $R x / P V X-C P$ were expressed in $N$. benthamiana, then $1 \mu \mathrm{M}$ fusicoccin was treated at 16 hr after infiltration.

\section{Confocal microscopy}

To measure $\mathrm{pH}$ on either side of the adjacent plasma membrane, two retiometirc pHluorin sensors, PMAPO and PM-CYTO were received from Martiniere et al. ${ }^{11}$. $N$. benthamiana leaves was infiltrated with a mixture of Agrobacteria harboring $\mathrm{pH}$ sensor and
XVE:CC ${ }^{A} 309$. Two days after infiltration, $10 \mu \mathrm{M} \beta$ estradiol solution was sprayed on Agrobacteriuminfiltrated region to activate $\mathrm{CC}^{\mathrm{A}} 309$ expression. Confocal microscopic observation and quantification of intensity of fluorescence signals were followed as described with modifications. Observations were performed with a Leica SP8 X microscope, using a $20 \mathrm{X}$ water objective with the same WLL laser at $20 \%$ of $476 \mathrm{~nm}$ and at $20 \%$ of $496 \mathrm{~nm}$ output. Emission light was at a pinhole of 1 Airy between 505 and 550 nm. For $\operatorname{DiBAC}_{4}(3)$ imaging, leaf discs were incubated in $\mathrm{DiBAC}_{4}(3)(10 \mu \mathrm{M}$, Invitrogen B438) for $30 \mathrm{~min}$ and we used the excitation line at $488 \mathrm{~nm}$ and recovered fluorescence signal between 505 and 560 $\mathrm{nm}$. Calibration curves were obtained in situ with the purified proteins from E. coli diluted in buffers of various pH levels. Yellow Cameleon 3.6 (YC3.6) was expressed in the same way as $\mathrm{pH}$ sensors. The $405 \mathrm{~nm}$ of a diode laser was used to excite CFP and FRET signal was collected in 525-535 $\mathrm{nm}$. To quantify the intensity of the fluorescence signal, images were analyzed using Image J software. After the subtraction of background noise, the average mean gray value was calculated for each channel and ratio images were generated.

\section{Statistics}

Graphs were generated by PRISM 7 (graphPad) and figure legends indicate type of statistical test used. Error bars generally represent standard deviation or standard error.

\section{References}

1. Maekawa, T., Kufer, T. A. \& Schulze-Lefert, P. NLR functions in plant and animal immune systems: so far and yet so close. Nat Immunol 12, 818-826, (2011).

2. Jones, J. D., Vance, R. E., Dangl, J. L. Intracellular innate immune surveillance devices in plants and animals. Science 354, (2016).

3. Dodds, P. N., Rathjen, J. P. Plant immunity: towards an integrated view of plant-pathogen interactions. Nat Rev Genet 11, 539-548, (2010).

4. Horsefield, S. et al. $\mathrm{NAD}^{+}$cleavage activity by animal an plant TIR domains in cell death pathways. Science 365, 793-799 (2019).

5. Wan, L. et al. TIR domains of plant immune receptors are $\mathrm{NAD}^{+}$-cleaving enzymes that promote cell death. Science 365, 799-803 (2019).

6. Wang, J. et al. Reconstitution and structure of a plant NLR resistosome conferring immunity. Science 364, doi:10.1126/science.aav5870 (2019). 
7. Lee, H. et al. Genome-wide functional analysis of hot pepper immune receptors reveals an autonomous NLR clade in seed plants. New Phytologist, https://doi.org/10.1111/nph.16878 (2020).

8. Haruta, M. et al. Molecular characterization of mutant Arabidopsis plants with reduced plasma membrane proton pump activity. $J$ Biol Chem 285, 17918-17929, (2010).

9. $\quad$ Serrano, R., Kiellandbrandt, M. C., Fink, G. R. Yeast plasma-membrane atpase is essential for growth and has homology with $\left(\mathrm{Na}^{+}+\mathrm{K}^{+}\right), \mathrm{K}^{+}$- and $\mathrm{Ca}^{2+}$-atpases. Nature 319, 689-693, (1986).

10. Bombarely, A. et al. A draft genome sequence of Nicotiana benthamiana to enhance molecular plant-microbe biology research. Mol Plant Microbe Interact 25, 1523-1530, (2012).

11. Martiniere, A. et al. Uncovering $\mathrm{pH}$ at both sides of the root plasma membrane interface using noninvasive imaging. P Natl Acad Sci USA 115, 6488-6493, (2018).

12. Lamb, C., Dixon, R. A. The Oxidative Burst in Plant Disease Resistance. Annu Rev Plant Physiol Plant Mol Biol 48, 251-275, (1997).

13. Grant, M. et al. The RPM1 plant disease resistance gene facilitates a rapid and sustained increase in cytosolic calcium that is necessary for the oxidative burst and hypersensitive cell death. Plant $J$ 23, 441450, (2000).

14. Nagai, T., Yamada, S., Tominaga, T., Ichikawa, M. \& Miyawaki, A. Expanded dynamic range of fluorescent indicators for $\mathrm{Ca}^{2+}$ by circularly permuted yellow fluorescent proteins. Proc Natl Acad Sci U S A 101, 10554-10559, (2004).

15. Konrad, K. R. \& Hedrich, R. The use of voltage-sensitive dyes to monitor signalinduced changes in membrane potentialABA triggered membrane depolarization in guard cells. Plant $J$ 55, 161-173, (2008).

16. Jelich-Ottmann, C., Weiler, E. W., Oecking, C. Binding of regulatory 14-3-3 proteins to the $\mathrm{C}$ terminus of the plant plasma membrane $\mathrm{H}+$-ATPase involves part of its autoinhibitory region. Journal of Biological Chemistry 276, 39852-39857, (2001).

17. Elmore, J. M., Coaker, G. The role of the plasma membrane $\mathrm{H}^{+}$-ATPase in plantmicrobe interactions. Molecular Plant 4, 416-427, (2011).

18. Baunsgaard, L. et al. The 14-3-3 proteins associate with the plant plasma membrane $\mathrm{H}^{+}$-ATPase to generate a fusicoccin binding complex and a fusicoccin responsive system. Plant J 13, 661-671, (1998).
19. Hamel, L. P. et al. The chloroplastic protein THF1 interacts with the coiled-coil domain of the disease resistance protein $\mathrm{N}^{\prime}$ and regulates light-dependent cell death. Plant Physiology 171, 658-674, (2016).

20. Kim, S. B. et al. The coiled-coil and leucinerich repeat domain of the potyvirus resistance protein Pvr4 has a distinct role in signaling and pathogen recognition. Mol Plant Microbe In 31, 906-913, (2018).

21. Harant, A., Sakai, T., Kamoun, S., Adachi, H. A vector system for fast-forward in vivo studies of the ZAR1 resistosome in the model plant Nicotiana benthamiana. bioRxiv, doi: https://doi.org/10.1101/2020.05.15.097584 (2020).

22. Jeworutzki, E. et al. Early signaling through the Arabidopsis pattern recognition receptors FLS2 and EFR involves $\mathrm{Ca}^{2+}$-associated opening of plasma membrane anion channels. Plant J 62, 367-378, (2010).

23. Sondergaard, T. E., Schulz, A., Palmgren, M. G. Energization of transport processes in plants. Roles of the plasma membrane $\mathrm{H}^{+}$ATPase. Plant Physiology 136, 2475-2482, (2004).

24 Oh, S. K., Kim, S. B., Yeom, S. I., Lee, H. A., Choi, D. Positive-selection and ligationindependent cloning vectors for large scale in planta expression for plant functional genomics. Mol Cells 30, 557-562, (2010).

25 Zuo, J., Niu, Q. W., Chua, N. H. Technical advance: an estrogen receptor-based transactivator XVE mediates highly inducible gene expression in transgenic plants. Plant J 24, 265-273, (2000).

26 Liu, Y., Schiff, M., Dinesh-Kumar, S. P. Virus-induced gene silencing in tomato. Plant J 31, 777-786, (2002).

27 Mang, $H$. et al. Differential regulation of two-tiered plant immunity and sexual reproduction by ANXUR receptor-like kinases. Plant Cell 29, 3140-3156, (2017).

28 Edgar, R. C. MUSCLE: multiple sequence alignment with high accuracy and high throughput. Nucleic Acids Res 32, 1792-1797, (2004).

29 Kumar, S., Stecher, G., Tamura, K. MEGA7: Molecular evolutionary genetics analysis version 7.0 for bigger datasets. Mol Biol Evol 33, 1870-1874, (2016).

\section{Acknowledgements}

We are thankful to Dr. Nadine Paris who provided pHluorin sensors and Dr. Cecile Segonzac for critical reading of the manuscript. This work was supported by the National Research Foundation of Korea (NRF) 
grant funded by the Korea government (MSIT) (No. 2018R1A5A1023599

(SRC)

2018R1A2A1A05019892).

\section{Author contributions}

D.C. conceived the project; H.L., H.M. Y.S., and D.C. designed the experiments. H.L., H.M., Y.S., J.L., S.O.,
S.L., J.K., S.J., H.K., H.P., S.K. performed the experiments. H.L., H.M., Y.S., and D.C. wrote the manuscript.

\section{Competing interest declaration}

Authors declare no competing interests. 


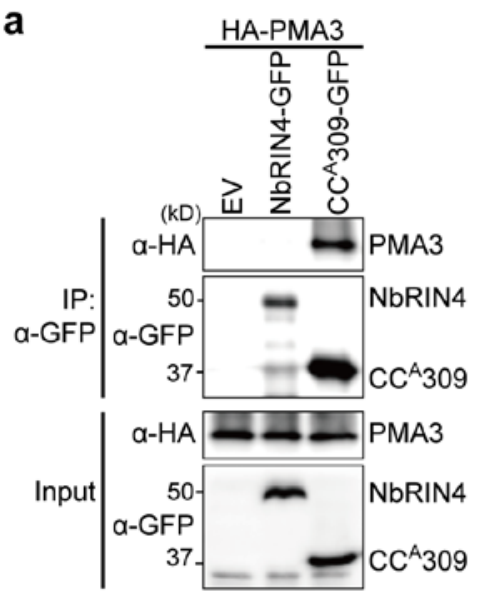

C

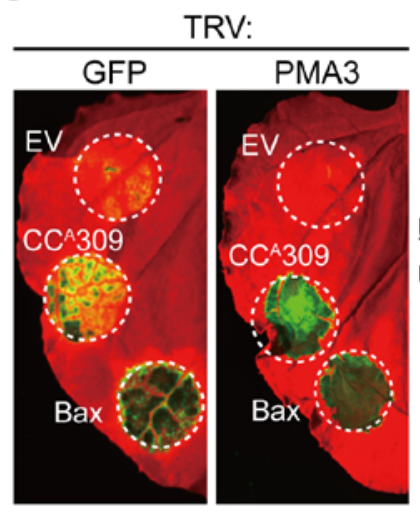

b

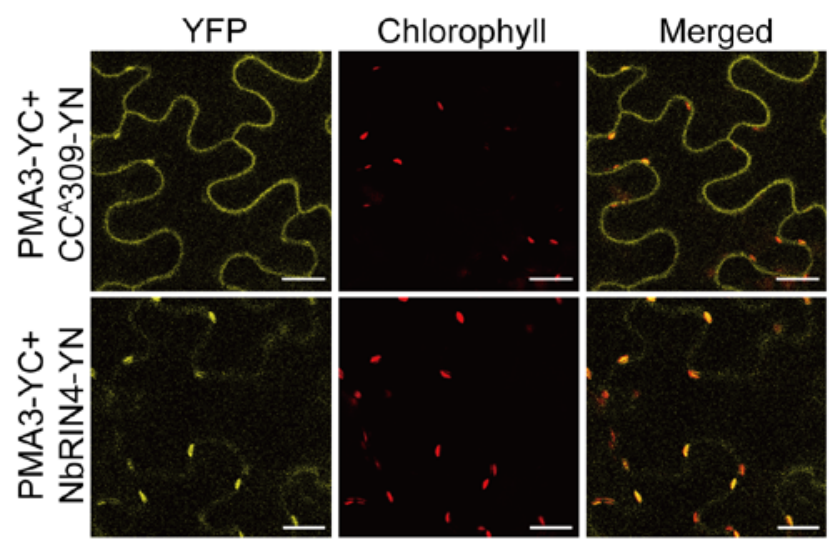

d
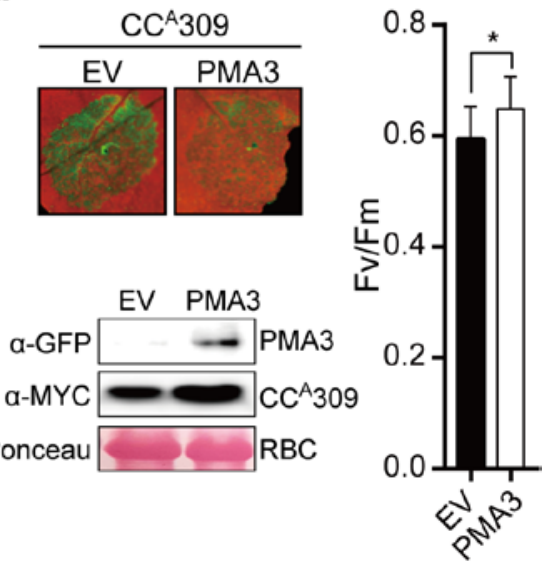

Fig. 1| The $\mathrm{CC}^{\mathrm{A}} 309$-induced cell death is negatively regulated by PMA3.

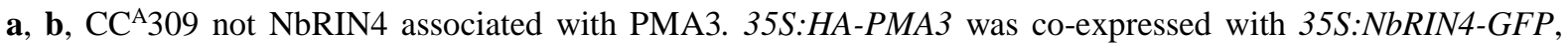
35S:CC $C^{A} 309-G F P$ or empty vector (EV) in N. benthamiana. Protein extracts were immunoprecipitated with GFP antibody (IP: $\alpha$-GFP) and immunoblotted with $\alpha$-GFP or $\alpha$-HA. Protein inputs are shown with immunoblotting before immunoprecipitation. a, BiFC assay in $N$. benthamiana. 35S:PMA3-YC was co-expressed with 35S:CC ${ }^{A} 309-Y N$ or 35S:NbRIN4-YN. b, The YFP signals were detected by a confocal microscope after 40 hours post agro-infiltration (hpi). YFP, yellow fluorescent protein signal; Chlorophyll, autofluorescence signal; Merged, YFP merged with Chlorophyll. Scale Bar $=20 \mu \mathrm{m}$. NbRIN4 localized in PM is used as a negative control. c, Enhanced CC ${ }^{\mathrm{A}} 309$-induced cell death in PMA3-silenced plants. Silenced plants by TRV:GFP or TRV:PMA3 were agro-infiltrated with $\mathrm{CC}^{\mathrm{A}} 309$ at three weeks after silencing. Leaves were photographed at 3-days post infiltration (dpi) (left panel) and the degree of cell death is depicted by quantification of quantum yield (Fv/Fm) (right panel). Human Bax and EV were used as positive and negative control for cell death, respectively. Significance was determined using t-test, with asterisks denoting statistically significant differences. ${ }^{* *} \mathrm{P}<0.01$. n.s.; no significant.

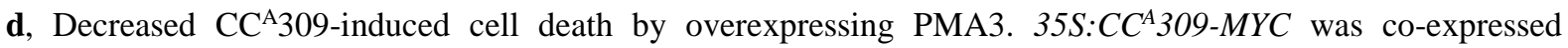
35S:PMA3-GFP or EV in N. benthamiana. Cell death was photographed at 5 dpi (left top) and quantified as Fv/Fm (right). The proteins were detected by immunoblotting with $\alpha$-GFP or $\alpha$-MYC. Ponceau S staining of RuBisCO (RBC) is loading control (left bottom). Data are represented as mean \pm SD $(\mathrm{N}=9)$ by triplicated independent experiments. Asterisk denotes a significant difference (t-test, $* \mathrm{P}<0.05)$. 
a

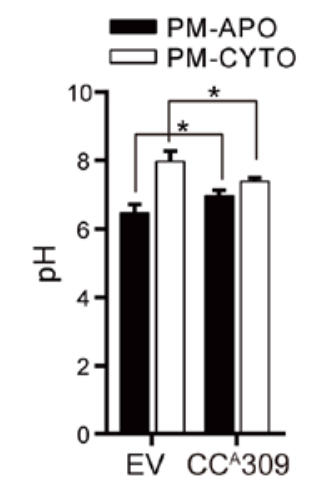

C
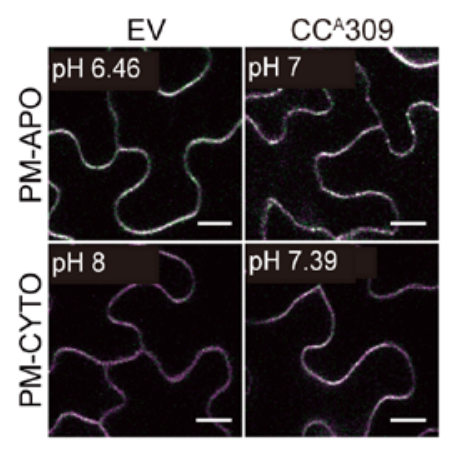

d

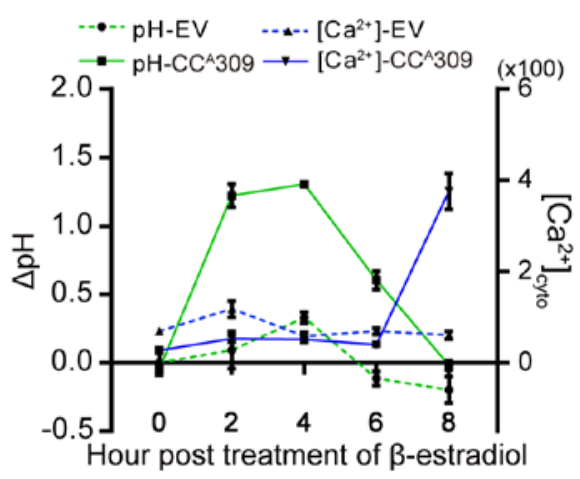

b
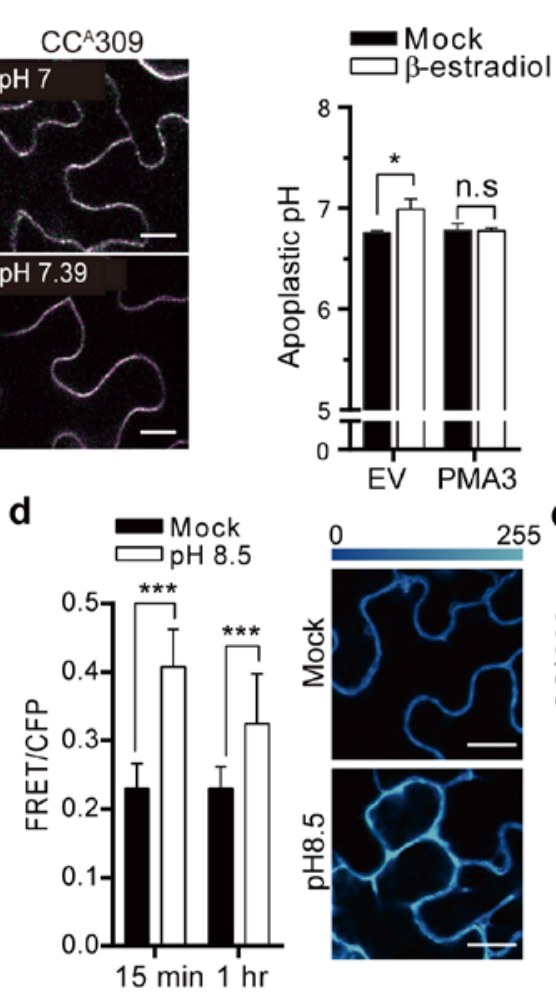



mStrawberry

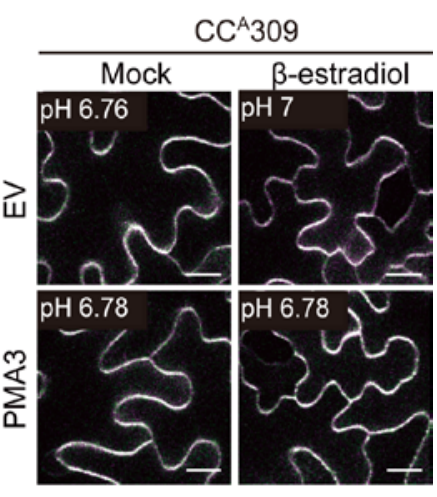

DiBAC4
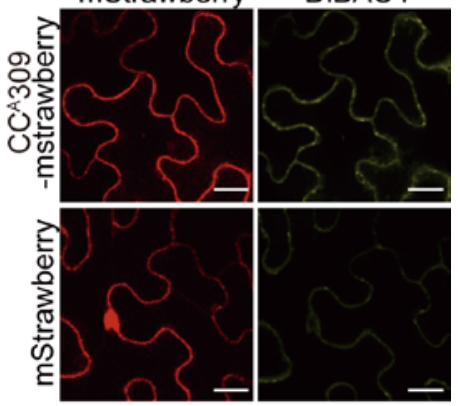

Fig. 2| $\mathrm{CC}^{\mathrm{A}} 309$ triggers an extracellular alkalization leading to cytosolic $\mathrm{Ca}^{2+}$ influx.

a, The elevated apoplastic $\mathrm{pH}$ and decreased cytoplasmic $\mathrm{pH}$ during $\mathrm{CC}^{\mathrm{A}} 309$-induced cell death in N. benthamiana . Beta-estradiol inducible $X V E: C C^{A} 309$ or EV were co-expressed with apoplastic (PM-APO) or cytoplasmic (PMCYTO) $\mathrm{pH}$ sensors. Bar $=15 \mu \mathrm{m}$. Data are represented as mean \pm SD by triplicated independent experiments. Asterisk denotes a significant difference (t-test, $\left.{ }^{*} \mathrm{P}<0.05\right)$. b. The compromised $\mathrm{CC}^{\mathrm{A}} 309$-induced apoplastic alkalization by PMA3. PM-APO was co-expressed with EV or 35S:PMA3-FLAG. The pH values (left) and representative confocal images (right) were taken $4 \mathrm{hr}$ after induction. Data are represented as mean \pm SD by triplicated independent experiments. Asterisk denotes a significant difference (t-test, $* \mathrm{P}<0.05)$. Bar $=15 \mu \mathrm{m}$. $\mathbf{c}$, The change of apoplastic $\mathrm{pH}$ and cytoplasmic $\mathrm{Ca}^{2+}$ accumulation. PM-APO or cytosolic $\mathrm{Ca}^{2+}$ sensor YC3.6 was co-expressed with EV or XVE:CC $C^{A} 309$. Apoplastic $\mathrm{pH}$ and cytosolic $\mathrm{Ca}^{2+}$ concentration was measured at indicated time points. d, Induced cytosolic $\mathrm{Ca}^{2+}$ accumulation by apoplastic alkalization in YC3.6 infiltrated plant. $10 \mathrm{mM}$ HEPES pH 7.0 (Mock) or pH 8.5 was infiltrated at 2 dpi. The $\mathrm{Ca}^{2+}$ concentration (left) and representative confocal images (right) were taken at $15 \mathrm{~min}$ and $1 \mathrm{hpi}$. Bar $=15 \mu \mathrm{m}$. e, CC ${ }^{\mathrm{A}} 309$-induced depolarization of plasma

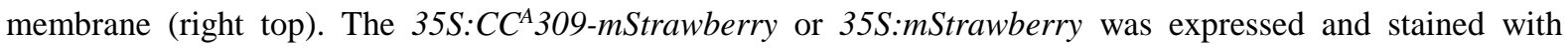
DiBAC4 at 8 hour post induction and photographed by confocal microscope. Bar $=20 \mu \mathrm{m}$. All experiment performed with 4-week-old $N$. benthamiana. Data are represented as mean $\pm \mathrm{SD}(\mathrm{N}=10)$ by triplicated independent experiments. Asterisk denotes a significant difference (t-test, $* * * \mathrm{P}<0.001)$. 


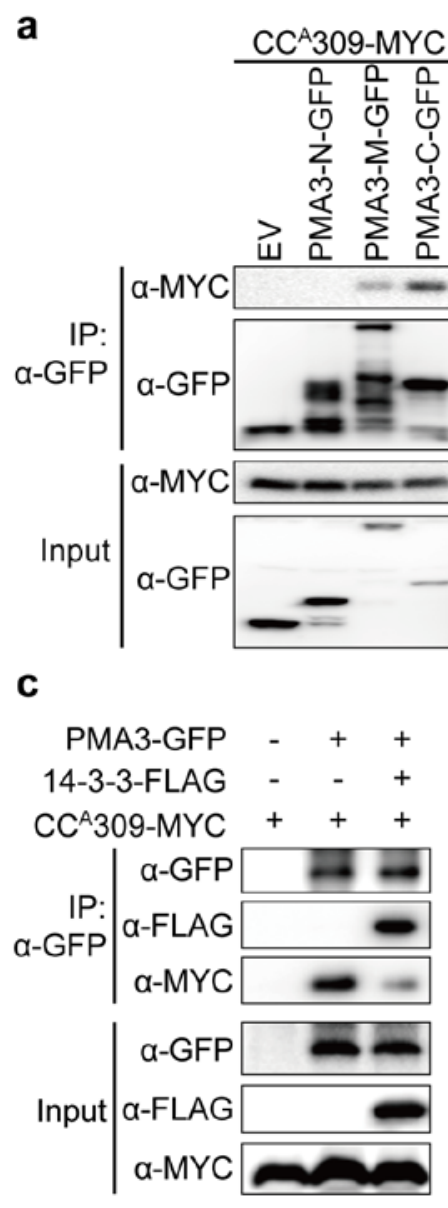

b

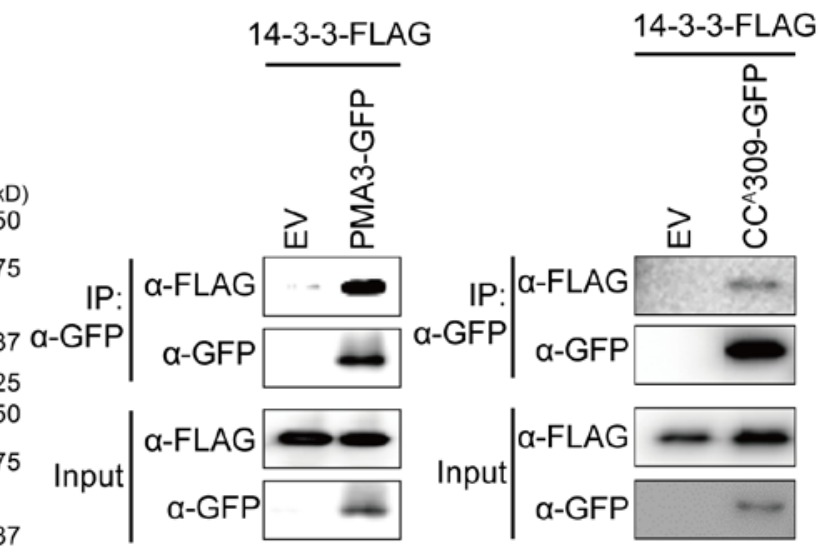

d

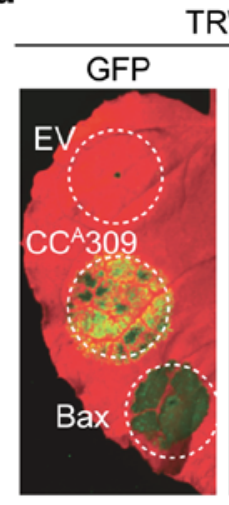

TRV:

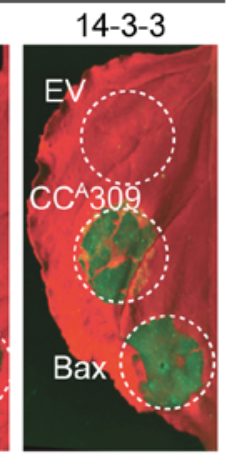

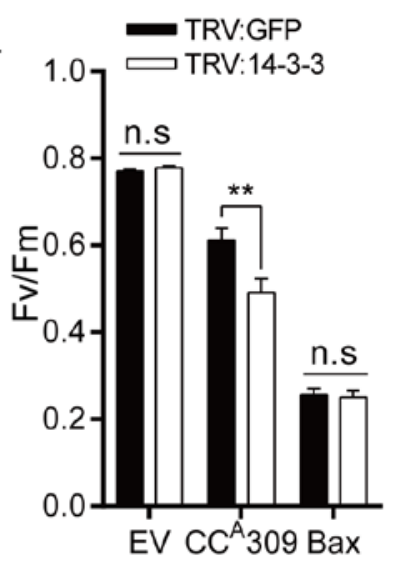

Fig. 3| 14-3-3 negatively regulates $\mathrm{CC}^{\mathrm{A}} 309$-induced cell death.

a, CC ${ }^{\mathrm{A}} 309$ interacts with cytosolic central domain (PMA3-M) and C-terminal domain (PMA3-C), but not Nterminal domain (PMA3-N) of PMA3. XVE:CC ${ }^{A} 309-M Y C$ was co-expressed with EV, 35S:PMA3-N-GFP, 35S:PMA3-M-GFP, or 35S:PMA3-C-GFP. Proteins extracts were immunoprecipitated with $\alpha$-GFP (IP: $\alpha-G F P)$ and immunoblotted with $\alpha$-MYC or $\alpha$-GFP (top two panel). Protein inputs are shown with immunoblotting before IP (bottom two panels). b, CC ${ }^{\mathrm{A}} 309$, PMA3, and 14-3-3 form complex. 35S:14-3-3-FLAG was co-expressed with EV and 35S:PMA3-GFP (left panel) or 35S:CC $309-G F P$ (right panel). Proteins extracts were immunoprecipitated with $\alpha$-GFP (IP: $\alpha$-GFP) and immunoblotted with $\alpha$-FLAG or $\alpha$-GFP (top two panel). Protein inputs are shown with immunoblotting before IP (bottom two panels). c, 14-3-3 inhibited the association between PMA3 and CC ${ }^{A} 309$. PMA3-GFP and/or CC ${ }^{A} 309-M Y C$ and/or EV were co-expressed with or without 14-3-3FLAG. The co-IP was carried out with an $\alpha$-GFP and analyzed by western blot with $\alpha$-GFP, $\alpha$-FLAG, or $\alpha$-MYC (left top three panels). Protein inputs are shown with immunoblotting before IP (left bottom three panels). d, The enhanced $C C^{A} 309$-induced cell death in 14-3-3-silenced plants. Three-week-old $N$. benthamiana silenced by TRV:GFP or TRV:14-3-3 were agro-infiltrated with EV, 35S:CCA309-FLAG, or 35S:Bax. The leaves were photographed at 5 dpi and the degree of cell death is depicted as quantum yield (Fv/Fm). The Data are shown as mean $\pm \mathrm{SE}(\mathrm{N}>12)$. Significance were determined using t-test, with asterisks denoting statistically significant differences. ${ }^{* *} \mathrm{P}<0.01$. 
a

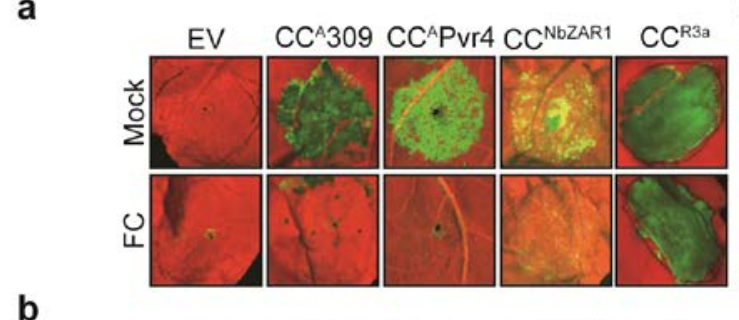

b

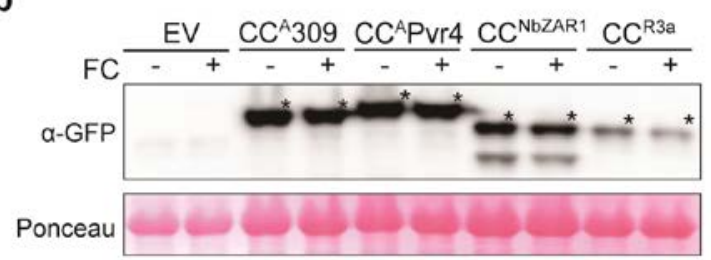

C

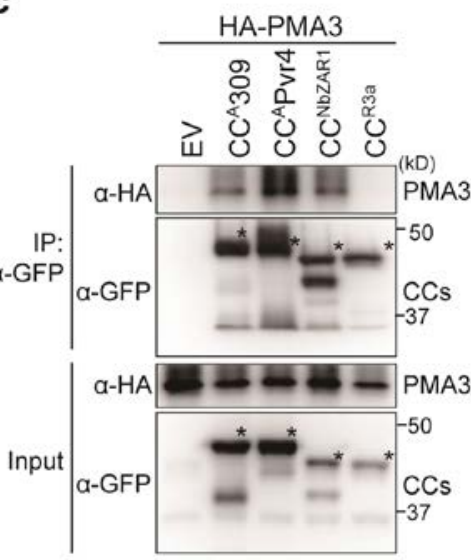

d

PM-localized CNL
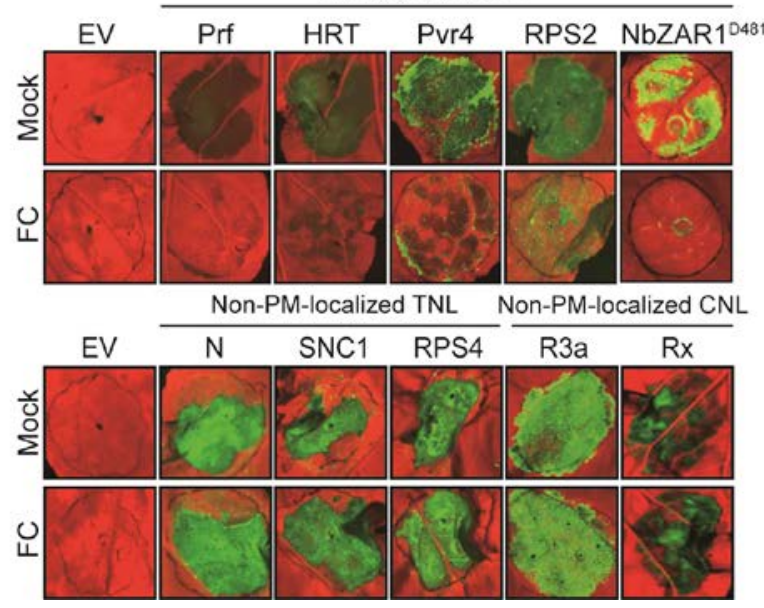

Fig. 4| The cell death induced by PM-localized CNLs is inhibited by PMA activator.

a, Fusicoccin (FC) inhibited cell death induced by CC domain of ANL309, Pvr4, NbZAR1, but not R3a. Agrobacterium carrying indicated construct were infiltrated in $N$. benthamiana then followed by infiltration of 1 $\mu \mathrm{M}$ fusicoccin at 16 hpi. Leaves were photographed at 3 dpi. b, FC did not affect the protein expression. The proteins were detected by immunoblotting with $\alpha$-GFP. Ponceau staining of RBC is loading control (bottom). c, The CC domains showing fusicoccin-dependent cell death inhibition are associated with PMA3. 35S:HA-PMA3 was co-expressed with CC domains. Proteins extracts were immunoprecipitated with $\alpha$-GFP (IP: $\alpha$-GFP) and immunoblotted with $\alpha$-HA. Protein inputs are shown with immunoblotting before IP. Asterisks indicate the expected protein bands. d, FC inhibited cell death mediated by PM-localized CNL-type R proteins, but not TNLs or other organelle-targeting CNLs. Fusicoccin was treated in the same way as (a) at the infiltrated region by Agrobacterium carrying R genes and cognate Avr genes. 


\section{Plant NLR targets P-type ATPase for executing plasma membrane depolarization leading to calcium influx and cell death}

Hye-Young Lee ${ }^{1} \dagger$, Ye-Eun Seo ${ }^{1,2} \dagger$, Joo Hyun Lee ${ }^{1}$, So Eui Lee ${ }^{1,2}$, Soohyun Oh ${ }^{1,2}$, Jihyun $\mathrm{Kim}^{1,2}$, Seungmee Jung ${ }^{1}$, Haeun Kim ${ }^{1,2}$, Hyojeong Park ${ }^{1,2}$, Sejun Kim ${ }^{1,2}$, Hyunggon Mang ${ }^{1 *}$, and Doil Choi ${ }^{1,2 *}$

Correspondence to: doil@snu.ac.kr (D.C.); skymang6@snu.ac.kr (H.M.) 
a

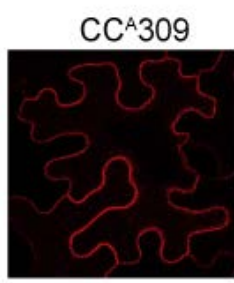

PMA3

Merged

b

NbRIN4
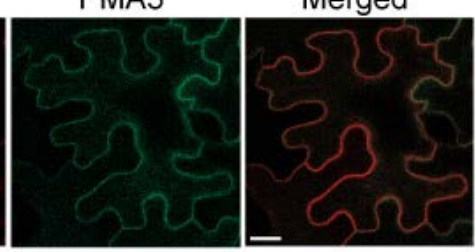

PMA3

Merged

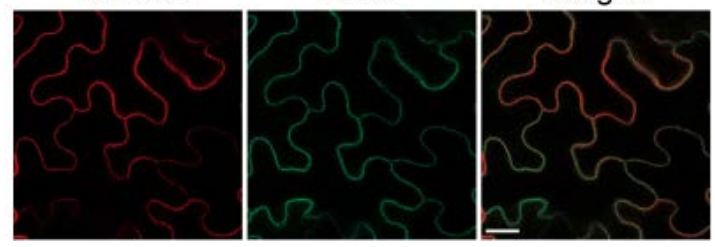

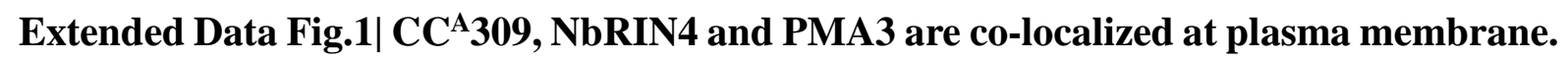

The subcellular localization of the CC $\mathrm{C}^{\mathrm{A}} 309$, NbRIN4, and PMA3 were determined in $N$. benthamiana epidermal cells. CC $^{\mathrm{A}} 309$, PMA3, and NbRIN4 localized in plasma membrane.

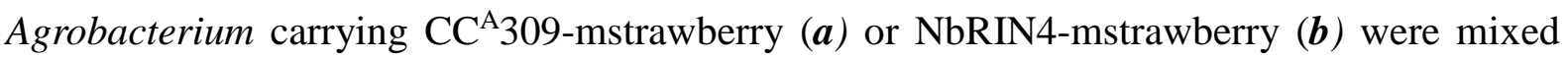
with PMA3-eGFP and infiltrated in 4 week-old $N$. benthamiana. At 2 dpi, the fluorescence signals were observed by confocal microscopy. The images were obtained by combination of Z-stack overlays. Bar $=20 \mu \mathrm{m}$. 

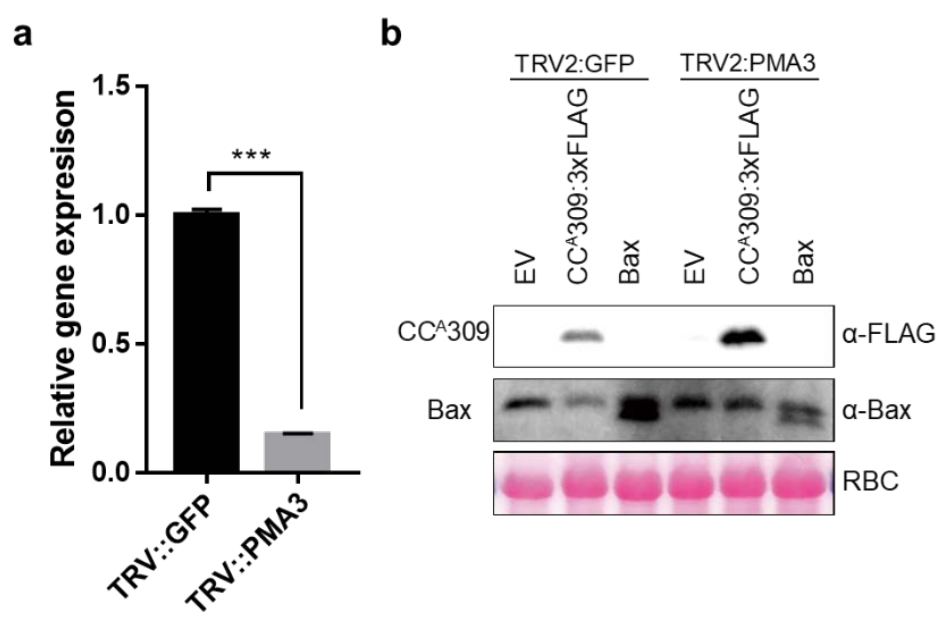

\section{Extended data Fig. 2| Virus-induced gene silencing of PMA3 and protein levels of overexpressed genes in PMA3-silenced plants.}

a, Decreased transcript of $P M A 3$ in $P M A 3$-silenced plant. The transcript level of $P M A 3$ was measured by quantitative RT-PCR at 2 weeks after VIGS. The mean values ( \pm SD) for transcript levels were normalized to that of $N$. benthamiana EF1- $\alpha$. Transcript levels PMA3 in GFPsilenced plants were set to 1 . Error bars represent the mean of three biological replicates. Asterisks denote significant differences at $* * * \mathrm{P}<0.001$ as determined by t-test. $\mathbf{b}$, Protein accumulation in GFP- or PMA3-silenced plants was confirmed by immunoblot analysis. Equal protein loading was confirmed by Ponceau staining of membrane. Asterisks indicate the expected protein bands. 


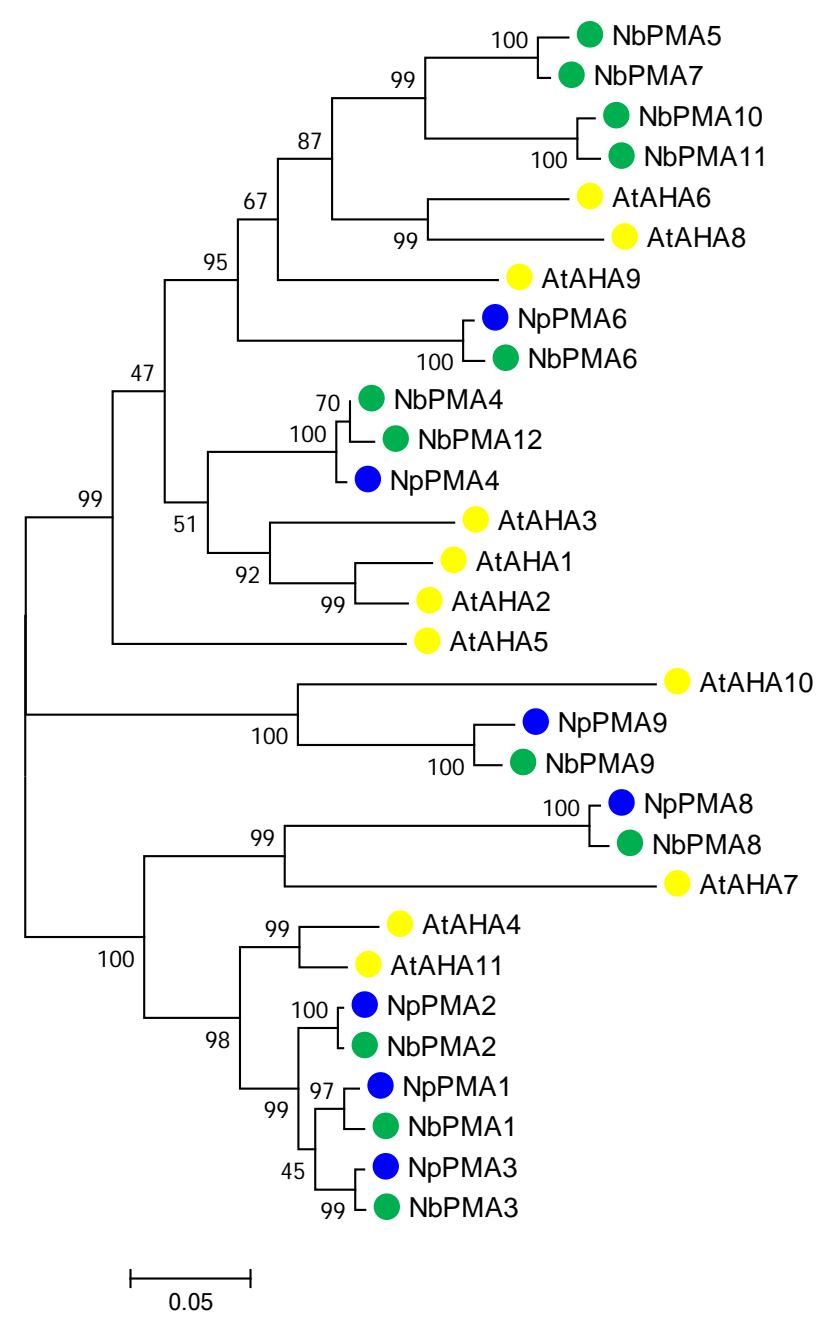

Extended Data Fig. 3| Phylogenetic analysis of PMAs in Arabidopsis, N. benthamiana and N. plumbaginifolia.

Amino acid sequences of 11 PMAs in Arabidopsis thaliana, 12 PMAs in N. benthamiana and 9 PMAs N. plumbaginifolia were aligned for construction of phylogenetic tree. Yellow; Arabidopsis PMAs; blue, N. plumbaginifolia; green, $N$. benthamiana. Phylogenetic analysis was conducted by a maximum likelihood method with 500 replications of bootsrapping in MEGA7. 


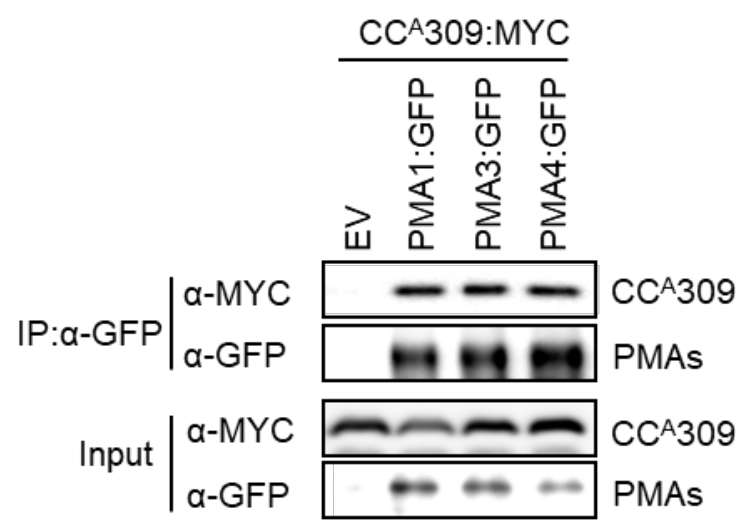

Extended Data Fig 4| PMA1 and PMA4 as well as PMA3 interact with CC ${ }^{\mathrm{A}} 309$.

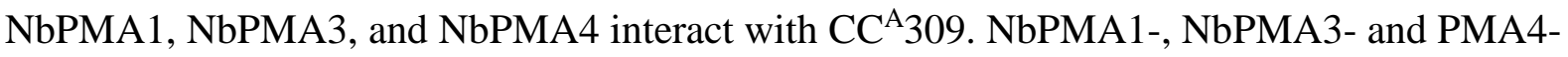
GFP were co-expressed with $\mathrm{CC}^{\mathrm{A}} 309-\mathrm{MYC}$ in $N$. benthamiana. Protein extracts were immunoprecipitaed with GFP antibody (IP: $\alpha$-GFP) and immunoblotted with $\alpha$-GFP or $\alpha$-MYC (top two panels). Protein inputs are shown with immunoblotting before immunoprecipitation (bottom two panels). 
a

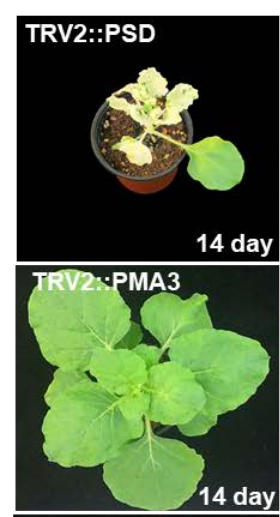

TRV2::PMA1/2/3/4

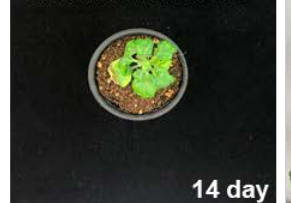

b

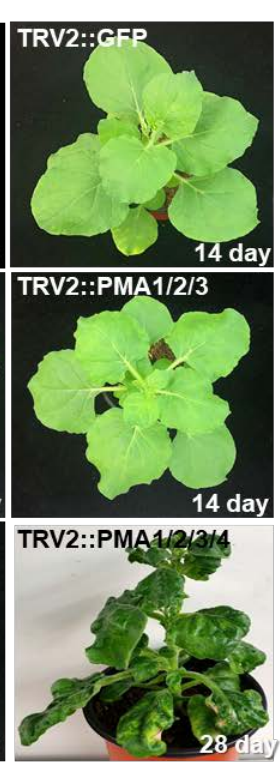

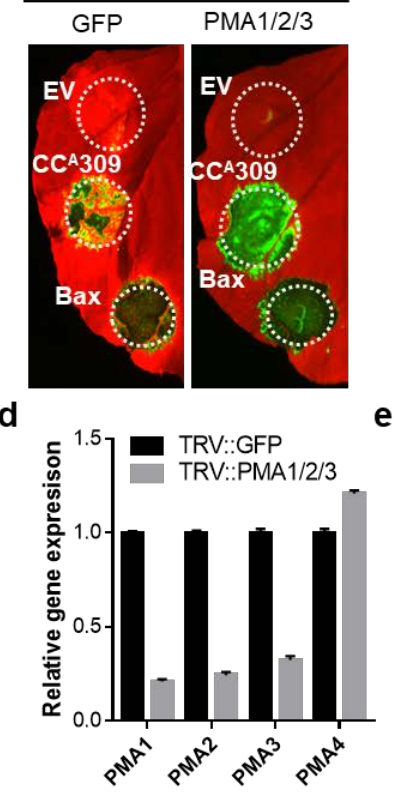

C

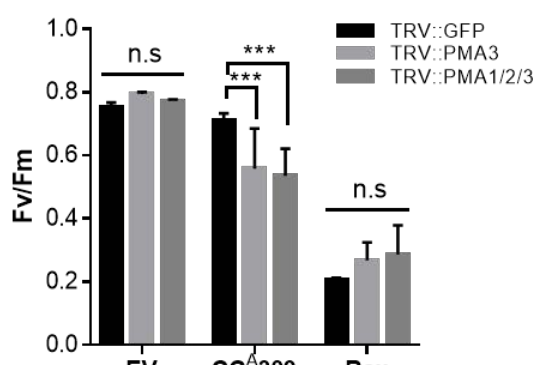

e

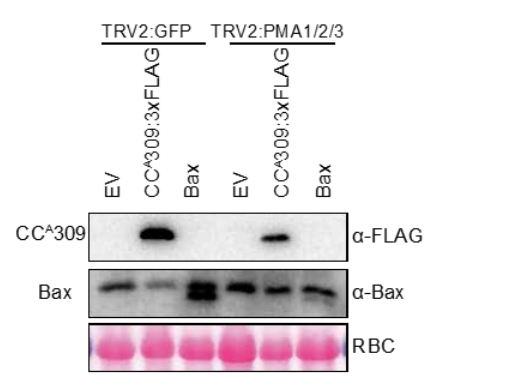

\section{Extended Data Fig 5| CC ${ }^{\mathrm{A}} 309$-induced cell death is enhanced in PMAs-silenced plants.}

a, The phenotypes of $N b P M A 3-, N b P M A 1 / 2 / 3-$ or $N b P M A 1 / 2 / 3 / 4$-silenced plants. Abnormal growth phenotypes were observed in $P M A 1 / 2 / 3 / 4$-silenced plant. The photobleached leaves of phytoene desaturase (PDS)-silenced plant represent the effective gene silencing. GFP-silenced plant was used as control for VIGS. Pictures were taken at 14 or 28 days after VIGS. b, The

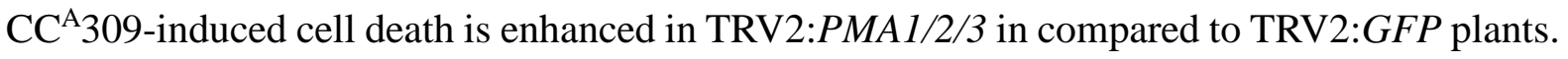
The plants at 10 days after VIGS were used to be infiltrated with Agrobacteria harboring EV, CC $^{\text {A } 309-3 x F L A G ~ a n d ~ B a x . ~ T h e ~ l e a v e s ~ w e r e ~ p h o t o g r a p h e d ~ a t ~} 3$ dpi. c, The data represents the degree of cell death using the quantum yield (Fv/Fm). Data are represented as mean $\pm \mathrm{SD}(\mathrm{n}=$ 9). d, The transcripts level of NbPMA1, NbPMA2, NbPMA3 and NbPMA4 in PMA1/2/3silenced plants were measured to estimate the silencing efficiency by qRT-PCR. The mean values $( \pm \mathrm{SD}$ ) for transcript levels were normalized to that of $N$. benthamiana EF1- $\alpha$. e, Protein accumulation of $\mathrm{CC}^{\mathrm{A}} 309$ and Bax in GFP- or NbPMA1/2/3-silenced plants was confirmed by immunoblot analysis. Protein loading was confirmed by Ponceau staining of membrane. Asterisks indicate the expected protein bands. 
a

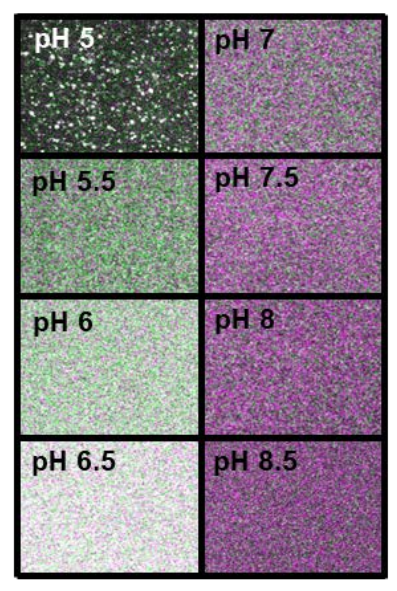

b

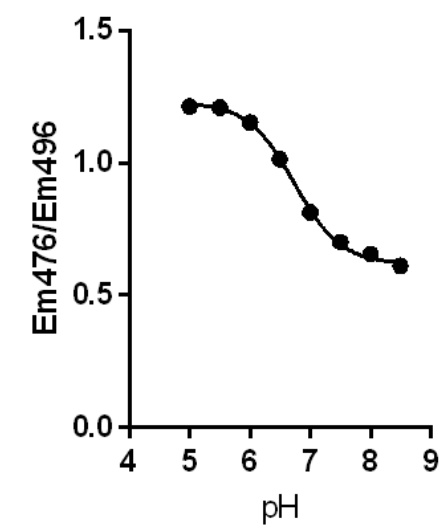

\section{Extended Data Fig 6| pHluorin response to external pH and calibration curve.}

a, Pseudocolored fluorescence images of pHluorin in buffers with different $\mathrm{pH}$ values. The purified pHluorin proteins from $E$. coli liquid culture were diluted in $50 \mathrm{mM}$ MES-KOH buffers at $\mathrm{pH} 5,5.5,6,6.5$ or in $50 \mathrm{mM}$ HEPES-KOH at $\mathrm{pH}$ 7, 7.5, 8, 8.5. The buffers containing the pHluorin dropped onto slide glass and illuminated at $497 \mathrm{~nm}$ and $496 \mathrm{~nm}$ using a confocal microscope. b, Calibration curve was obtained from (a) to define absolute $\mathrm{pH}$ values. 


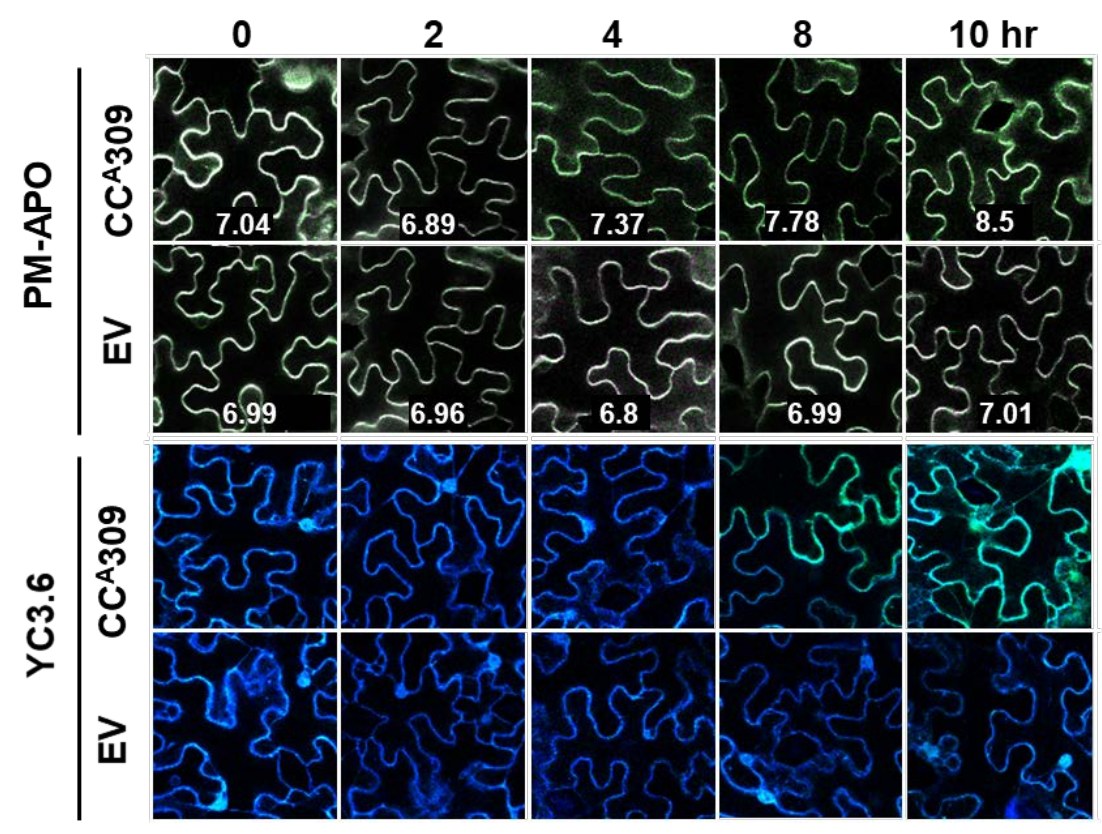

Extended Data Fig 7| The earlier alkalizations in apoplast then cytosolic $\mathrm{Ca}^{2+}$ accumulation during $\mathrm{CC}^{\mathrm{A}} 309$ expression.

Pseudocolored fluorescence images of PM-APO and YC3.6 were obtained from transiently expressed leaves infiltrated with EV or inducible $\mathrm{CC}^{\mathrm{A}} 309$ and PM-APO or YC3.6 in $N$. benthamiana. respectively. Pictures were taken at various time points after spray of $10 \mu \mathrm{M}$ of $\beta$-estratiol solution to induce $\mathrm{CC}^{\mathrm{A}} 309$. 


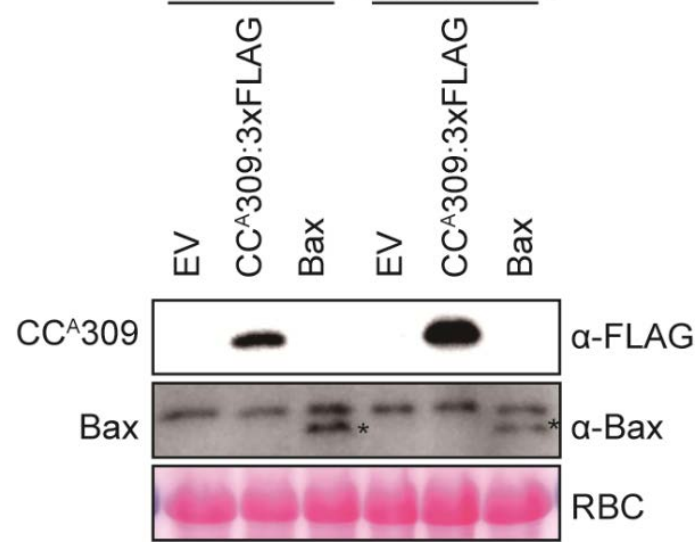

Extended Data Fig 8| Protein accumulation of $\mathrm{CC}^{\mathrm{A}} 309$ and Bax in GFP- or Nb14-3-3silenced plants.

Protein accumulation of $\mathrm{CC}^{\mathrm{A}} 309$ and Bax in GFP- or 14-3-3-silenced plants was confirmed by immunoblot analysis with $\alpha$-FLAG or $\alpha$-Bax antibody (top two panels). Equal protein loading was confirmed by Ponceau staining of membrane (bottom panel). Asterisks indicate the expected protein bands. 




b

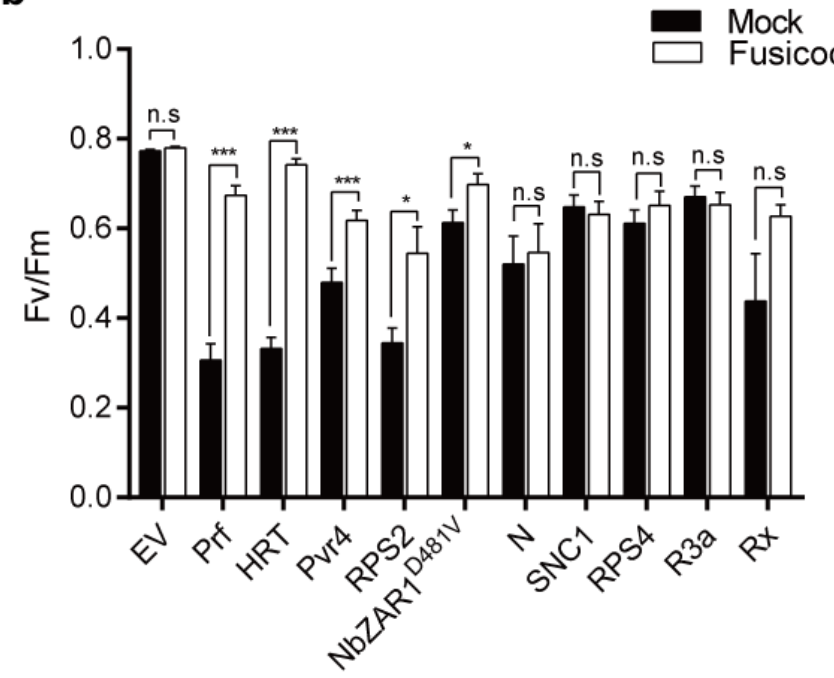

Extended Data Fig 9| The cell death induced by CC domains or R proteins was inhibited by treatment of fusicoccin.

$\boldsymbol{a}, \boldsymbol{b}$, The degree of cell death in Fig. 4a, d is depicted by quantification of quantum yield (Fv/Fm). Data are represented as mean \pm standard error $(n=5 \sim 25)$ by triplicated independent experiments. Significance was determined using t-test. $*<0.05$, ${ }^{* *} \mathrm{p}<0.01, * * * \mathrm{P}<0.001$. 


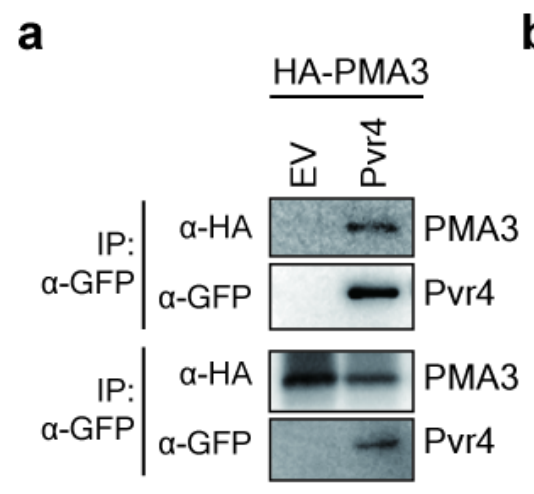

b

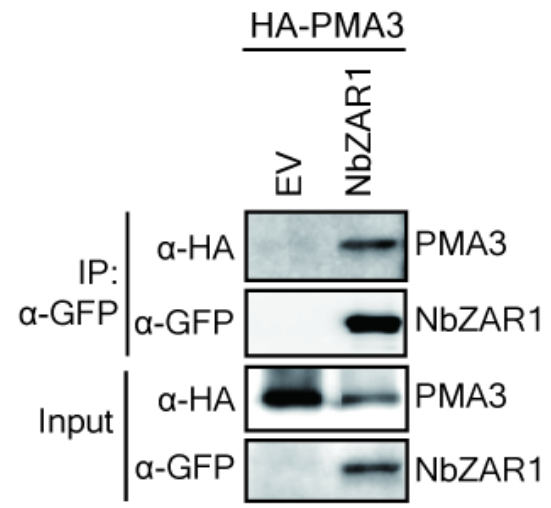

Extended Data Fig 10| Full-length R proteins, Pvr4 and NbZAR1 also interact with PMA3.

35S:HA-PMA3 was co-expressed with EV and 35S:Pvr4-GFP $a$ or 35S:NbZAR1-GFP $b$. Proteins extracts were immunoprecipitated with $\alpha$-GFP (IP: $\alpha$-GFP) and immunoblotted with $\alpha$-HA or $\alpha$-GFP (top two panel). Protein inputs are shown with immunoblotting before IP (bottom two panels). 

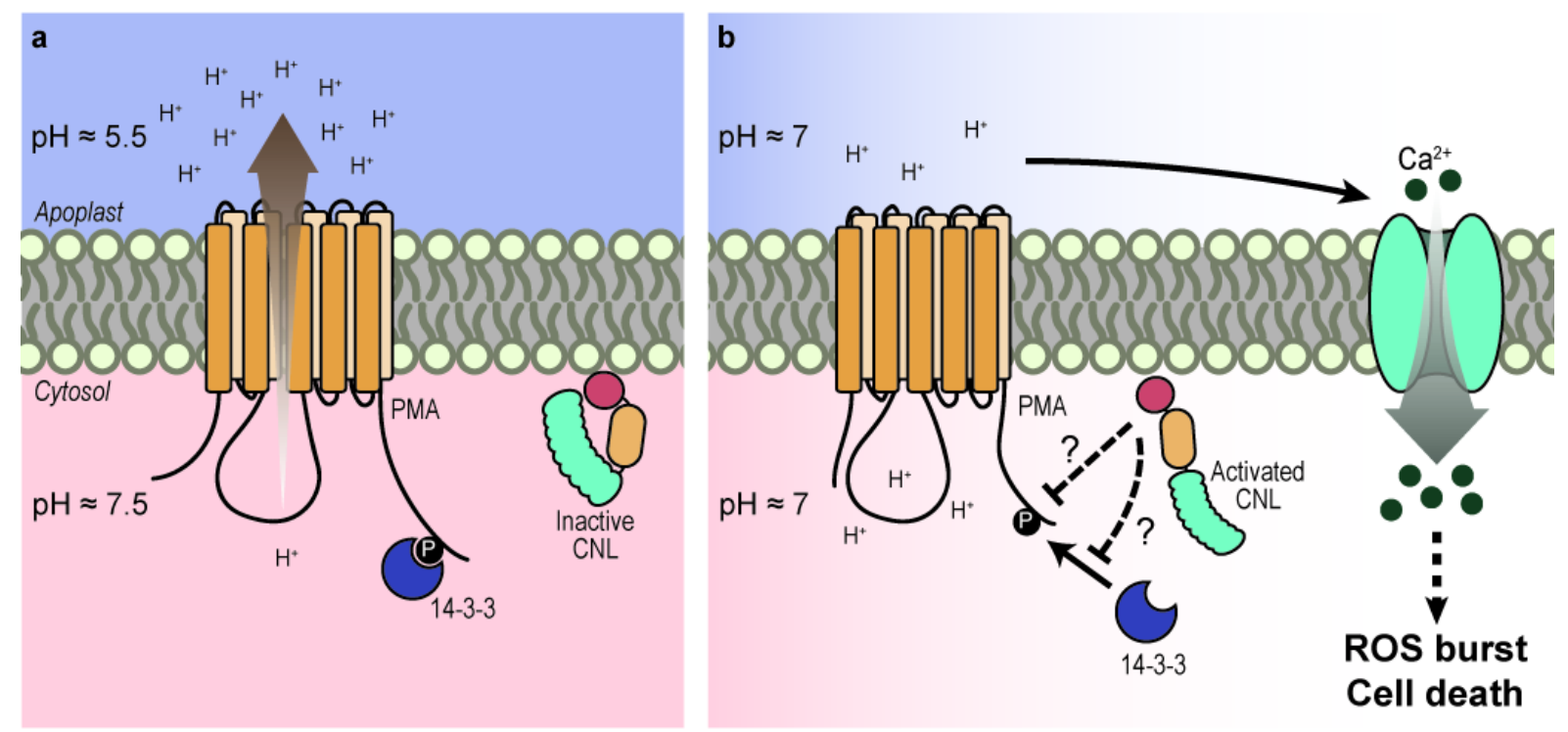

Extended Data Fig 11| Proposed mechanism of plasma membrane-associated CNLmediated cell death.

a, The rest state of polarized plasma membrane. b, PM depolarization triggered by activated CNLs. The change of PM potential might facilitate a series of PM-integrated defense responses such as $\mathrm{Ca}^{2+}$ influx and ROS burst, and consequently leads to cell death. 


\section{Extended table 1| List of the $\mathrm{CC}^{\mathrm{A}} 309$ interactor candidates identified by mass spectrometry}

\begin{tabular}{|c|c|c|c|c|}
\hline \multirow[b]{2}{*}{ Gene description } & \multirow[b]{2}{*}{ Gene ID } & \multicolumn{2}{|c|}{ \# of peptides } & \multirow[b]{2}{*}{$\begin{array}{l}\text { Co- } \\
\text { IP }\end{array}$} \\
\hline & & $\begin{array}{l}\text { 35S:GFP- } \\
\text { 3xFLAG }\end{array}$ & $\begin{array}{l}\text { 35S:CC }{ }^{A} 309- \\
\text { 3xFLAG }\end{array}$ & \\
\hline $\mathrm{CC}^{\mathrm{A}} 309$ & & 0 & 223 & \\
\hline GFP & & 1352 & 0 & \\
\hline ATP synthase epsilon chain & Niben101Scf01399g00012.1 & 2 & 30 & + \\
\hline Acetyl-CoA carboxylase & Niben101Scf04077g03006.1 & 0 & 26 & - \\
\hline Cell division protein FtsZ homolog 1 & Niben101Scf03107g01011.1 & 0 & 24 & n.t \\
\hline Chaperonin 60 & Niben101Scf02842g00014.1 & 0 & 21 & - \\
\hline Glutathione S-transferase & Niben101Scf08250g03016.1 & 1 & 21 & n.t \\
\hline EPSIN2 & Niben101Scf01066g01009.1 & 2 & 18 & n.t \\
\hline Peptidyl-prolyl cis-trans isomerase FKBP16-3 & Niben101Scf06423g01016.1 & 0 & 18 & + \\
\hline Cinnamoyl-CoA reductase 2-like & Niben101Scf09883g01010.1 & 0 & 16 & - \\
\hline Acyl-CoA-binding protein & Niben101Scf02240g02008.1 & 0 & 13 & n.t \\
\hline Thioredoxin superfamily protein & Niben101Scf10490g00011.1 & 0 & 12 & - \\
\hline Thiamine pyrophosphate enzyme & Niben101Scf02538g06008.1 & 0 & 11 & - \\
\hline Coiled coil domain containing protein & Niben101Scf10747g00018.1 & 0 & 10 & n.t \\
\hline Chaperonin 60-2 & Niben101Scf29076g00011.1 & 0 & 10 & - \\
\hline Macrophage migration inhibitory factor & Niben101Scf00302g02011.1 & 0 & 10 & - \\
\hline Reactive Intermediate Deaminase A & Niben101Scf04339g07004.1 & 0 & 9 & + \\
\hline ATP synthase delta chain & Niben101Scf15372g00003.1 & 0 & 8 & - \\
\hline Translation elongation factor EF1B & Niben101Scf03350g00002.1 & 0 & 7 & - \\
\hline Trigalactosyldiacylglycerol 2 & Niben101Scf07377g00001.1 & 0 & 7 & + \\
\hline pre-mRNA-processing factor 39 & Niben101Scf11535g01033.1 & 0 & 7 & n.t \\
\hline 2-methylene-furan-3-one reductase & Niben101Scf01155g00008.1 & 0 & 7 & - \\
\hline Plasma membrane ATPase 3 & Niben101Scf07395g00031.1 & 0 & 6 & + \\
\hline Peroxiredoxin & Niben101Scf03671g02013.1 & 0 & 6 & - \\
\hline Superoxide dismutase & Niben101Scf04451g00026.1 & 0 & 5 & - \\
\hline Tripeptidyl-peptidase 2 isoform & Niben101Scf00595g08041.1 & 0 & 5 & n.t \\
\hline Light-harvesting complex II proteins & Niben101Scf06008g00011.1 & 0 & 4 & + \\
\hline $\begin{array}{l}\text { Zinc finger A20 and AN1 domain-containing } \\
\text { protein }\end{array}$ & Niben101Scf05348g04001.1 & 0 & 3 & - \\
\hline
\end{tabular}


Extended table $2 \mid$ List of primers used in this study

\begin{tabular}{|c|c|c|c|}
\hline Name & Primer (5'-3') & Description & Ref \\
\hline CC $^{\mathrm{A}} 309-\mathrm{AF}$ & $\begin{array}{l}\text { CGACGACAAGACCCTATGGCTGTGG } \\
\text { AAATCATAACTGC }\end{array}$ & $\begin{array}{l}\text { For overexpression of CCA309 with C- } \\
\text { terminal epitope tag }\end{array}$ & \\
\hline CC ${ }^{A} 309-A R$ & $\begin{array}{l}\text { GAGGAGAAGAGCCCTAACCGGATA } \\
\text { GCAGAAAACAGC }\end{array}$ & $\begin{array}{l}\text { For overexpression of CCA309 with C- } \\
\text { terminal epitope tag }\end{array}$ & \\
\hline NbRIN4_AF & $\begin{array}{l}\text { CGACGACAAGACCCTATGGCACGTC } \\
\text { CAAATGTTCCGAAG }\end{array}$ & $\begin{array}{l}\text { For overexpression of NbRIN4 with C- } \\
\text { terminal epitope tag }\end{array}$ & \\
\hline NbRIN4-AR & $\begin{array}{l}\text { GAGGAGAAGAGCCCTCCAGGGACA } \\
\text { GCAGCACTTCATC }\end{array}$ & $\begin{array}{l}\text { For overexpression of NbRIN4 with C- } \\
\text { terminal epitope tag }\end{array}$ & \\
\hline NbPMA3-AF & $\begin{array}{l}\text { CGACGACAAGACCCTATGGGAGAG } \\
\text { AAGCCTGAAGTATTG }\end{array}$ & $\begin{array}{l}\text { For overexpression of NbPMA3 with C- } \\
\text { terminal epitope tag }\end{array}$ & \\
\hline NbPMA3-AR & $\begin{array}{l}\text { GAGGAGAAGAGCCCTAACGGTGTAT } \\
\text { GACTGCTGAATTGTC }\end{array}$ & $\begin{array}{l}\text { For overexpression of NbPMA3 with C- } \\
\text { terminal epitope tag }\end{array}$ & \\
\hline PMA3-N-AF & $\begin{array}{l}\text { CGACGACAAGACCCTATGGGAGAG } \\
\text { AAGCCTGAAGTATTG }\end{array}$ & $\begin{array}{l}\text { For overexpression of } \mathrm{N} \text {-terminal domain of } \\
\text { PMA3 with epitope }\end{array}$ & \\
\hline PMA3-N-AR & $\begin{array}{l}\text { GAGGAGAAGAGCCCTTTTCAAGAAT } \\
\text { TTGCTCTCСТTCTTCTCС }\end{array}$ & $\begin{array}{l}\text { For overexpression of } \mathrm{N} \text {-terminal domain of } \\
\text { PMA3 with epitope }\end{array}$ & \\
\hline PMA3-M-AF & $\begin{array}{l}\text { CGACGACAAGACCCTATGTCTCATC } \\
\text { GTCTTGCTCAACAGGGC }\end{array}$ & $\begin{array}{l}\text { For overexpression of central domain of } \\
\text { PMA3 with epitope }\end{array}$ & \\
\hline PMA3-M-AR & $\begin{array}{l}\text { GAGGAGAAGAGCCCTCGTGTAGTTT } \\
\text { TTCATCCTTTGAAAGATTGC }\end{array}$ & $\begin{array}{l}\text { For overexpression of central domain of } \\
\text { PMA3 with epitope }\end{array}$ & \\
\hline PMA3-C-AF & $\begin{array}{l}\text { CGACGACAAGACCCTATGAAGTTCT } \\
\text { TCATCCGTTATGCTCTCAGTG }\end{array}$ & $\begin{array}{l}\text { For overexpression of C-terminal domain of } \\
\text { PMA3 with epitope }\end{array}$ & \\
\hline PMA3-C-AR & $\begin{array}{l}\text { GAGGAGAAGAGCCCTAACGGTGTAT } \\
\text { GACTGCTGAATTGTCTC }\end{array}$ & $\begin{array}{l}\text { For overexpression of C-terminal domain of } \\
\text { PMA3 with epitope }\end{array}$ & \\
\hline Nb14-3-3_AF & $\begin{array}{l}\text { CGACGACAAGACCCTATGGCGGTGG } \\
\text { CACCGACG }\end{array}$ & $\begin{array}{l}\text { For overexpression of } 14-3-3 \text { with C- } \\
\text { terminal epitope tag }\end{array}$ & \\
\hline Nb14-3-3_AR & $\begin{array}{l}\text { GAGGAGAAGAGCCCTATTTTTGGCT } \\
\text { TCATCAGGTTTGGG }\end{array}$ & $\begin{array}{l}\text { For overexpression of } 14-3-3 \text { with C- } \\
\text { terminal epitope tag }\end{array}$ & \\
\hline Pvr4-CC-AF & $\begin{array}{l}\text { ATGGAAATCGTAACTGCTATTTTGA } \\
\text { GC }\end{array}$ & $\begin{array}{l}\text { For overexpression of CC domain of Pvr } 4 \\
\text { with C-terminal epitope tag }\end{array}$ & \\
\hline Pvr4-CC-AR & $\begin{array}{l}\text { GAGGAGAAGAGCCCTCAAAGCTGTC } \\
\text { ATGACCTCTTCC }\end{array}$ & $\begin{array}{l}\text { For overexpression of CC domain of Pvr4 } \\
\text { with C-terminal epitope tag }\end{array}$ & \\
\hline NbZAR1-AF & $\begin{array}{l}\text { CGACGACAAGACCCTATGGTGGATG } \\
\text { CGGTGGTCACTG }\end{array}$ & $\begin{array}{l}\text { For overexpression of NbZAR1 with C- } \\
\text { terminal epitope tag }\end{array}$ & 21 \\
\hline NbZar1-AR & $\begin{array}{l}\text { GAGGAGAAGAGCCCTGTTCCTATGT } \\
\text { TCTTCCTTCTTCCATAGACC }\end{array}$ & $\begin{array}{l}\text { For overexpression of NbZAR1 with C- } \\
\text { terminal epitope tag }\end{array}$ & 21 \\
\hline NbZar1-CC-AR & $\begin{array}{l}\text { GAGGAGAAGAGCCCTATTGTGTGCC } \\
\text { TCCATACTTCCTTCT }\end{array}$ & $\begin{array}{l}\text { For overexpression of CC domain of } \\
\text { NbZAR1 with C-terminal epitope tag }\end{array}$ & 21 \\
\hline AVR3a_KI-AF & $\begin{array}{l}\text { CGACGACAAGACCCTATGGACCAAA } \\
\text { CCAAGGTCCTGG }\end{array}$ & $\begin{array}{l}\text { For overexpression of Avr3a with C- } \\
\text { terminal epitope tag }\end{array}$ & \\
\hline AVR3a-AR & $\begin{array}{l}\text { GAGGAGAAGAGCCCTCATGCATTCC } \\
\text { CTATCGATCTTTATG }\end{array}$ & $\begin{array}{l}\text { For overexpression of Avr3a with C- } \\
\text { terminal epitope tag }\end{array}$ & \\
\hline R3a-CC-AF & $\begin{array}{l}\text { CGACGACAAGACCCTATGGAGATTG } \\
\text { GCTTAGCAGTTGG }\end{array}$ & $\begin{array}{l}\text { For overexpression of CC domain of R3a } \\
\text { with C-terminal epitope tag }\end{array}$ & \\
\hline R3a-CC-AF & $\begin{array}{l}\text { GAGGAGAAGAGCCCTAGTCGAACC } \\
\text { AAAATGTTCCTTTAAGCC }\end{array}$ & $\begin{array}{l}\text { For overexpression of CC domain of R3a } \\
\text { with C-terminal epitope tag }\end{array}$ & \\
\hline NbPMA1-AF & $\begin{array}{l}\text { CGACGACAAGACCCTATGGGGGAG } \\
\text { GAGAAGCCTG }\end{array}$ & $\begin{array}{l}\text { For overexpression of NbPMA1 with C- } \\
\text { terminal epitope tag }\end{array}$ & \\
\hline NbPMA1-AR & $\begin{array}{l}\text { GAGGAGAAGAGCCCTAACAGTGTAT } \\
\text { GCCTGTTGAATTGTC }\end{array}$ & $\begin{array}{l}\text { For overexpression of NbPMA1 with C- } \\
\text { terminal epitope tag }\end{array}$ & \\
\hline NbPMA2-AF & $\begin{array}{l}\text { CGACGACAAGACCCTATGGGGGAG } \\
\text { AAACCTGAAGTG }\end{array}$ & $\begin{array}{l}\text { For overexpression of NbPMA2 with C- } \\
\text { terminal epitope tag }\end{array}$ & \\
\hline NbPMA2-AR & $\begin{array}{l}\text { GAGGAGAAGAGCCCTAACAGTGTAT } \\
\text { GATTGCTGAATTGTCTC }\end{array}$ & $\begin{array}{l}\text { For overexpression of NbPMA2 with C- } \\
\text { terminal epitope tag }\end{array}$ & \\
\hline NbPMA4-AF & $\begin{array}{l}\text { CGACGACAAGACCCTATGGCAAAAG } \\
\text { CTATCAGCCTCG }\end{array}$ & $\begin{array}{l}\text { For overexpression of NbPMA4 with C- } \\
\text { terminal epitope tag }\end{array}$ & \\
\hline NbPMA4-AR & $\begin{array}{l}\text { GAGGAGAAGAGCCCTAACTGTATAA } \\
\text { TGCTGCTGGATCG }\end{array}$ & $\begin{array}{l}\text { For overexpression of NbPMA4 with C- } \\
\text { terminal epitope tag }\end{array}$ & \\
\hline YN_F & $\begin{array}{l}\text { GCTCTTCTCCTCAGAGAGCAAAAGT } \\
\text { TGATTTCTGAGGAGG }\end{array}$ & For construction of Bi-FC vector & \\
\hline $\begin{array}{l}\text { YN_mid R (PstI } \\
\text { site removal) }\end{array}$ & TTGCAGGCCGTAGCCGAAG & For construction of Bi-FC vector & \\
\hline
\end{tabular}




\begin{tabular}{|c|c|c|}
\hline $\begin{array}{l}\text { YN_mid Forward } \\
\text { (PstI site removal) }\end{array}$ & $\begin{array}{l}\text { TACGGCCTGCAATGCTTCGCCCGCT } \\
\text { ACCC }\end{array}$ & For construction of Bi-FC vector \\
\hline YN_AR & GAGGAGAAGAGCCCTTTAGGCCATG & For construction of Bi-FC vector \\
\hline YC_F & $\begin{array}{l}\text { GCTCTTCTCCTCATGTACCCATACGA } \\
\text { TGTTCCAGATTACG }\end{array}$ & For construction of Bi-FC vector \\
\hline YC_AR & $\begin{array}{l}\text { GAGGAGAAGAGCCCTTTACTTGTAC } \\
\text { AGCTCGTCCATGCC }\end{array}$ & For construction of Bi-FC vector \\
\hline $\begin{array}{l}\text { VIGS-NbPMA3- } \\
\text { AF }\end{array}$ & $\begin{array}{l}\text { CGACGACAAGACCCTTCATTACATT } \\
\text { GCTTATAATCAACTCCAC }\end{array}$ & For NbPMA3 silencing construct \\
\hline $\begin{array}{l}\text { VIGS-NbPMA3- } \\
\text { AR }\end{array}$ & $\begin{array}{l}\text { GAGGAGAAGAGCCCTGATTATGTCC } \\
\text { CCAGGCACGAG }\end{array}$ & For NbPMA3 silencing construct \\
\hline $\begin{array}{l}\text { VIGS- } \\
\text { VIPMA1/2/3-AF }\end{array}$ & CGACGACAAGACCCTCCTTTCATGG & For NbPMA1/2/3 silencing construct \\
\hline $\begin{array}{l}\text { NbPMA1/2/3-AF } \\
\text { VIGS- }\end{array}$ & $\begin{array}{l}\text { TGCTTATCATTGCAATTCTTAAT } \\
\text { GAGGAGAAGAGCCCTATTGTAGAGC }\end{array}$ & For NbPMA1/2/3 silencing construct \\
\hline $\begin{array}{l}\text { NbPMA1/2/3-AR } \\
\text { TRV-PMA1_tR }\end{array}$ & $\begin{array}{l}\text { CAGATCACTCCAGC } \\
\text { GCTTCTCCTCCСССССАAAAATTTCA } \\
\text { AGAATTTGC }\end{array}$ & For NbPMA1/2/3/4 silencing construct \\
\hline TRV-PMA2_tF & $\begin{array}{l}\text { AAATTTTTGGGGTTTATGTGGAATCC } \\
\text { TCTCTCATGG }\end{array}$ & For NbPMA1/2/3/4 silencing construct \\
\hline TRV-PMA2_tR & GGAGCGAGCCGAGCCATG & For NbPMA1/2/3/4 silencing construct \\
\hline TRV-PMA3_tF & $\begin{array}{l}\text { TCGGCTCGCTCCGAAAGCCAAGGTC } \\
\text { CTTCGAG }\end{array}$ & For NbPMA1/2/3/4 silencing construct \\
\hline TRV-PMA3_tR & AGACACCATCTCCAGGACC & For NbPMA1/2/3/4 silencing construct \\
\hline TRV-PMA4_tF & $\begin{array}{l}\text { GAGATGGTGTCTTCTCTGGCTCAACT } \\
\text { TGCAAAC }\end{array}$ & For NbPMA1/2/3/4 silencing construct \\
\hline TRV-PMA4_AR & $\begin{array}{l}\text { GAGGAGAAGAGCCCTGTACATGACT } \\
\text { ATAATCTCAACGAGC }\end{array}$ & For NbPMA1/2/3/4 silencing construct \\
\hline TRV-14-3-3-AF & $\begin{array}{l}\text { CGACGACAAGACCCTGAGCAAGCTG } \\
\text { AGAGGTACGAAG }\end{array}$ & For 14-3-3 silencing construct \\
\hline TRV-14-3-3-AF & $\begin{array}{l}\text { GAGGAGAAGAGCCCTGATGGGATA } \\
\text { AGCTTTGCATCGAGC }\end{array}$ & For 14-3-3 silencing construct \\
\hline PMA1-qRT_F & GTCATTGCTCAACTGGTTGCA & For qRT PCR \\
\hline PMA1-qRT_R & $\begin{array}{l}\text { GATGATATCAAGAGGGATATAGAAT } \\
\text { ACTAG }\end{array}$ & For qRT PCR \\
\hline PMA2-qRT_F & GCCCATGCACAAAGGACT & For qRT PCR \\
\hline PMA2-qRT_R & CAGTGTATGCAGCTCACGTTGC & For qRT PCR \\
\hline PMA3-qRT_F & AGTGATCTGGCTCTACAATATTGTG & For qRT PCR \\
\hline PMA3-qRT_R & $\begin{array}{l}\text { CGCTGTTCTTTCCCGAAATCTTTTTT } \\
\mathrm{T}\end{array}$ & For qRT PCR \\
\hline PMA4_qRT_F & GCACTTGCTCAGAGAACAC & For qRT PCR \\
\hline PMA4_qRT_R & CTCAACGTGACCCTTGAGGGTA & For qRT PCR \\
\hline 14-3-3c-qRT_F & GCAAAGCTTATCCСТTCTGCAGC & $\begin{array}{l}\text { For qRT PCR } \\
\text { (Niben101Scf02537g00004.1) }\end{array}$ \\
\hline 14-3-3c-qRT_R & GAGAGTACTCTCAGCAGCTTCC & $\begin{array}{l}\text { For qRT PCR } \\
\text { (Niben101Scf02537g00004.1) }\end{array}$ \\
\hline 14-3-3c-qRT_F & $\begin{array}{l}\text { GCAAAGCTTATCCCATCTGCAGCAT } \\
\text { C }\end{array}$ & $\begin{array}{l}\text { For qRT PCR } \\
\text { (Niben101Scf02367g04001.1) }\end{array}$ \\
\hline 14-3-3c-qRT_R & GAGTGTACTCTCAGCAGCCTC & $\begin{array}{l}\text { For qRT PCR } \\
\text { (Niben101Scf02367g04001.1) }\end{array}$ \\
\hline
\end{tabular}

